\title{
TLR3 absence confers increased survival with improved macrophage activity against pneumonia
}

\author{
Madathilparambil V. Suresh, ${ }^{1}$ Vladislav A. Dolgachev, ${ }^{1}$ Boya Zhang, ${ }^{,}$Sanjay Balijepalli, ${ }^{1}$ \\ Samantha Swamy, ${ }^{1}$ Jashitha Mooliyil,, Georgia Kralovich, ${ }^{1}$ Bivin Thomas, ${ }^{1}$ David Machado-Aranda, \\ Monita Karmakar, ${ }^{1}$ Sanjeev Lalwani, ${ }^{2}$ Arulselvi Subramanian, ${ }^{2}$ Arun Anantharam, ${ }^{3}$ \\ Bethany B. Moore, ${ }^{4}$ and Krishnan Raghavendran ${ }^{1}$ \\ 'Department of Surgery, University of Michigan, Ann Arbor, Michigan, USA. ${ }^{2}$ Department of Laboratory Medicine, All India \\ Institute of Medical Sciences, New Delhi, India. ${ }^{3}$ Department of Pharmacology and ${ }^{4}$ Department of Medicine, University of \\ Michigan, Ann Arbor, Michigan, USA.
}

Toll-like receptor 3 (TLR3) is a pathogen recognition molecule associated with viral infection with double-stranded RNA (dsRNA) as its ligand. We evaluated the role of TLR3 in bacterial pneumonia

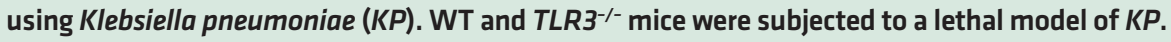
Alveolar macrophage polarization, bactericidal activity, and phagocytic capacity were compared. RNA-sequencing was performed on alveolar macrophages from the WT and $T L R^{-1-}$ mice. Adoptive transfers of alveolar macrophages from $T L R 3^{-/-}$mice to WT mice with $K P$ were evaluated for survival. Expression of TLR3 in postmortem human lung samples from patients who died from gram-negative pneumonia and pathological grading of pneumonitis was determined. Mortality was significantly lower in $\mathrm{TLR3}^{--}$, and survival improved in WT mice following antibody neutralization of TLR3 and with TLR3/dsRNA complex inhibitor. Alveolar macrophages from $\mathrm{TLR3}^{--}$mice demonstrated increased bactericidal and phagocytic capacity. RNA-sequencing showed an increased production of chemokines in $\mathrm{TLR}^{-/-}$mice. Adoptive transfer of alveolar macrophages from the $T L R 3^{-/-}$mice restored the survival in WT mice. Human lung samples demonstrated a good correlation between the grade of pneumonitis and TLR3 expression. These data represent a paradigm shift in understanding the mechanistic role of TLR3 in bacterial pneumonia.

Conflict of interest: The authors have declared that no conflict of interest exists.

Copyright: (c) 2019, American Society for Clinical Investigation.

Submitted: June 30, 2019

Accepted: October 17, 2019

Published: December 5, 2019.

Reference information: /CI Insight. 2019;4(23):e131195.

https://doi.org/10.1172/jici.

insight.131195.

\section{Introduction}

Bacterial pneumonia remains a major cause of morbidity and mortality in modern intensive care units (ICUs) (1). The incidence of ventilator-associated pneumonia (VAP) has steadily increased despite the introduction of the VAP bundle in the care of critically ill patients $(2,3)$. Lung contusion (LC) is the primary cause of mortality following blunt chest trauma and blast explosions, while being an independent risk factor for the development of acute respiratory distress syndrome and VAP (4). Klebsiella pneumoniae $(K P)$ is a capsulated gram-negative pathogen that causes a significant proportion of community-acquired and hospital-acquired pneumonia $(5,6)$. These organisms are particularly virulent as they are typically responsible for $70 \%$ of VAP cases (7). In addition, they can cause significant alveolar necrosis and potential septicemia. These conditions result in substantial mortality and morbidity.

Pattern recognition receptors (PRRs), such as those classified under the toll-like receptor (TLR) family, consisting of 10 highly homologous TLRs in humans, are involved in detection and response $(8,9)$, often acting through inflammatory pathways directed by NF-кB. TLRs in alveolar macrophages recognize the molecular constituents of pathogens and activate the host's innate immune responses (4). TLR3 is considered a pathogen recognition molecule associated with viral infections, with double-stranded RNA (dsRNA) serving as its sole ligand $(10,11)$. TLR3 activation stimulates the production of type I interferon (IFN) as the main mechanism of antiviral immunity. TLR3 is unique in its binding specificity to dsRNA. In addition, TLR3 activation promotes the systemic hyperinflammatory response observed during sepsis (12). We have recently demonstrated that TLR3, independent of a type I IFN response, also functions in initiation and maintenance of acute inflammatory responses following LC (4). Although these studies have indicated a role for TLR3, the precise nature 
of the ligand and the downstream signaling pathways involved are still unclear. Moreover, the specific role of TLR3 in lung contusion, bacterial pneumonia, and other nonviral diseases remains largely unknown.

Several earlier studies investigated the role of TLRs in host defense against $K P(13-15)$. Our laboratory recently reported on the role of TLR3 in the activation and progression of acute inflammation following blunt trauma-induced LCs. In this study, we use C57BL/6 and $T L R 3^{-1-}$ knockout mice to examine the role of TLR3 in the initiation and maintenance of primary and secondary bacterial pneumonia. Specifically, we explore the major mechanisms by which alveolar macrophages are involved in the processes related to diminished bacterial clearance observed with activation of TLR3. We hypothesize that dsRNA release from necrotic cells following bacterial pneumonia activates TLR3 located on alveolar macrophages, inducing apoptosis and phagosomal maturation arrest, thereby worsening acute inflammation and increasing bacterial presence. Our current results have the potential to change the paradigm for the role of TLR3 in bacterial infections.

\section{Results}

\section{Postmortem lung tissue from patients and mice infected with $K P$ shows significant expression of TLR3}

Human postmortem lung samples. We examined the expression of TLR3 using IHC from postmortem lung samples of patients who died from $K P$. Immunocytochemistry data showed that the lung samples from different $K P$ patients had significantly higher expression of TLR3 (Figure 1A). Histopathological evaluation of postmortem samples from patients with $K P$ revealed a significantly higher degree of pneumonitis characterized by the influx of macrophages compared with samples from normal lungs (Figure 1A). Additionally, we examined the blood and tracheal cultures of patients infected with other gram-negative bacteria. These data suggest that activation of TLR3 is also observed in other gram-negative bacterial infections, such as Pseudomonas and Acinetobacter infections (Tables 1-3). A Kendall's $\tau_{\mathrm{b}}$ correlation was used to determine the relationship between pneumonitis grading and the IHC scores among the 10 postmortem samples and showed that there was a good positive correlation between the two, which was statistically significant $\left(\tau_{\mathrm{b}}=0.6571\right.$, and $P=0.03$ ).

Murine lung samples. Here, we further examined the expression of TLR3 in WT mice following KP via immunocytochemistry. Immunocytochemistry images show intense TLR3 staining in alveolar macrophages following $K P$ inoculation compared with uninjured mice (Figure 1B).

Capillary Western blot. Additionally, lung protein expression of TLR3 was measured by Western blot following pneumonia. The protein levels of TLR3 expression were higher at 24 hours in the WT mice compared with the $T L R 3^{--}$mice following pneumonia (Figure 1C). Taken together with the previous immunocytochemistry imaging, these data corroborate the idea that there is greater activation of TLR3 following pneumonia.

TLR3 activation is lethal in models of primary and secondary bacterial pneumonia of $K P$ $T L R 3^{-1-}$ mice show reduced mortality in primary and secondary bacterial pneumonia with $K P$. We examined the protective effect of TLR3 knockout in mice following primary and secondary $K P$ infection. Following a primary insult of $K P$, all WT mice died by day 5 , but 19 of the $20 T L R 3^{-/-}$mice survived. This phenomenon was also observed in the setting of secondary bacterial pneumonia $(\mathrm{LC}+K P)$, in which mice were subjected to blunt force trauma as well as inoculation with $K P$, either concurrently or at 6 hours following LC (Figure 2, A and B).

The effect of monoclonal antibody neutralization against TLR3 before pneumonia. We previously found the extent of lung injury and inflammation following LC was significantly reduced in the $T L R 3^{-/-}$mice and WT mice treated with monoclonal antibody against TLR3 (4). In this study, we administered mouse monoclonal antibody against TLR3 (50 and $100 \mu \mathrm{g}$ /mouse) 48 hours before $K P$ infection in C57BL/ 6 mice while using IgG and saline for the control mice. As shown in Figure 2C, neutralization of TLR3 with antibody protected the mice following $K P$. These data demonstrate that the absence of TLR 3 confers a protective role, even though TLR3 has not been identified as a ligand for recognition of $K P$.

Reduced lung injury and inflammation in the $T L R 3^{-/-}$mice following primary and secondary pneumonia correlate with improved bacterial clearance

Lung injury and inflammation in TLR $3^{--}$and WT mice following KP administration. We performed several additional experiments using $T L R 3^{-/-}$and WT mice to examine the pathogenesis of acute inflammation and injury in primary $(K P)$ bacterial pneumonia. It is important to note that while the model of LC is nonlethal, the pneumonia model by itself is lethal $(5,16)$. 
A

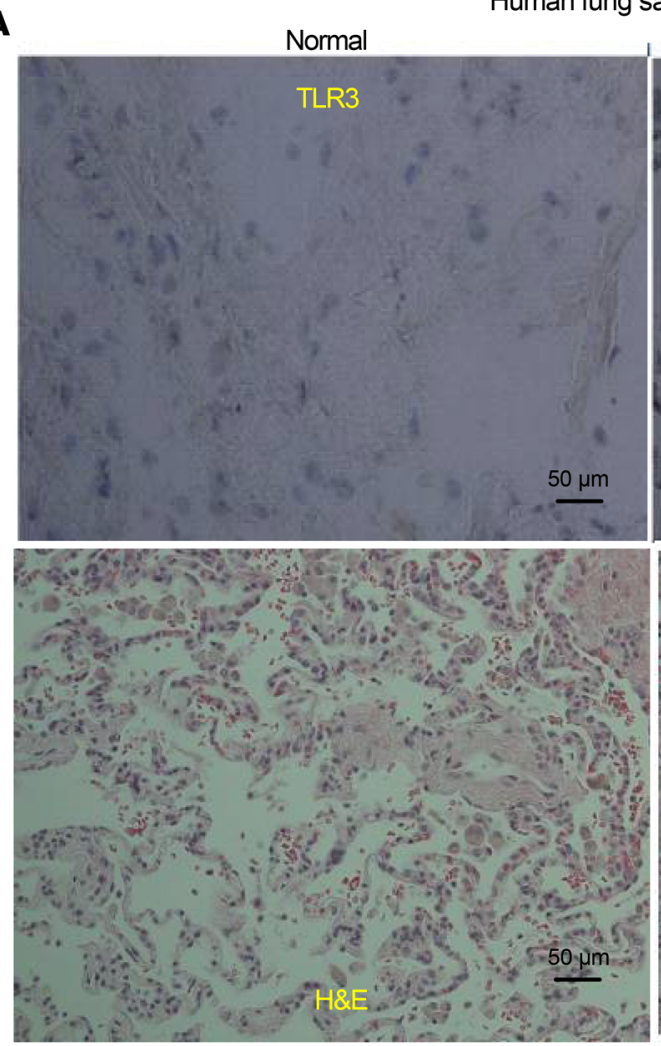

Human lung samples
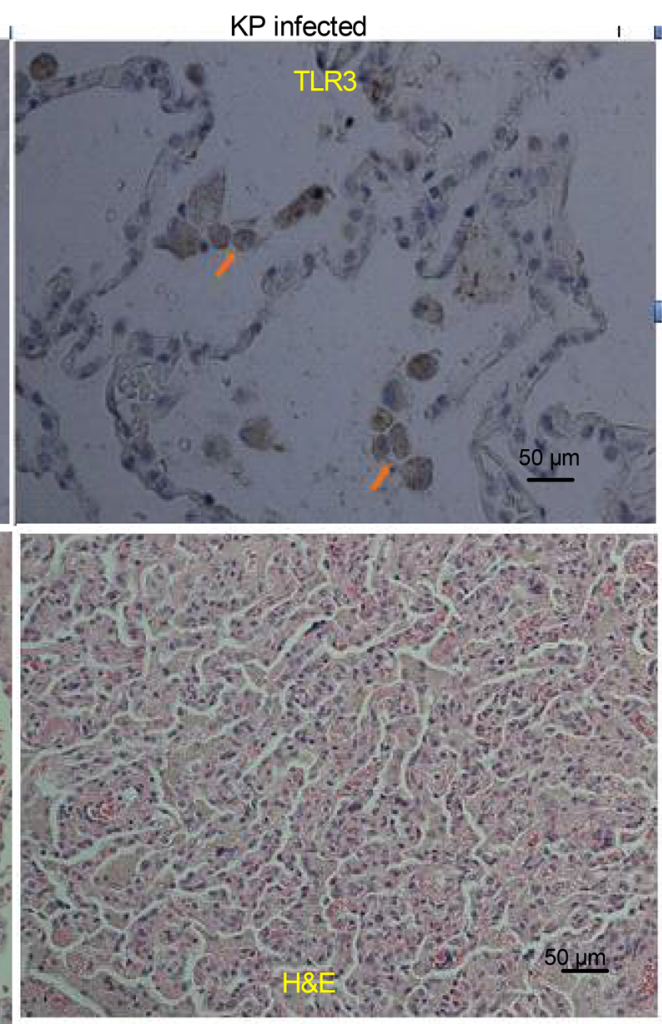
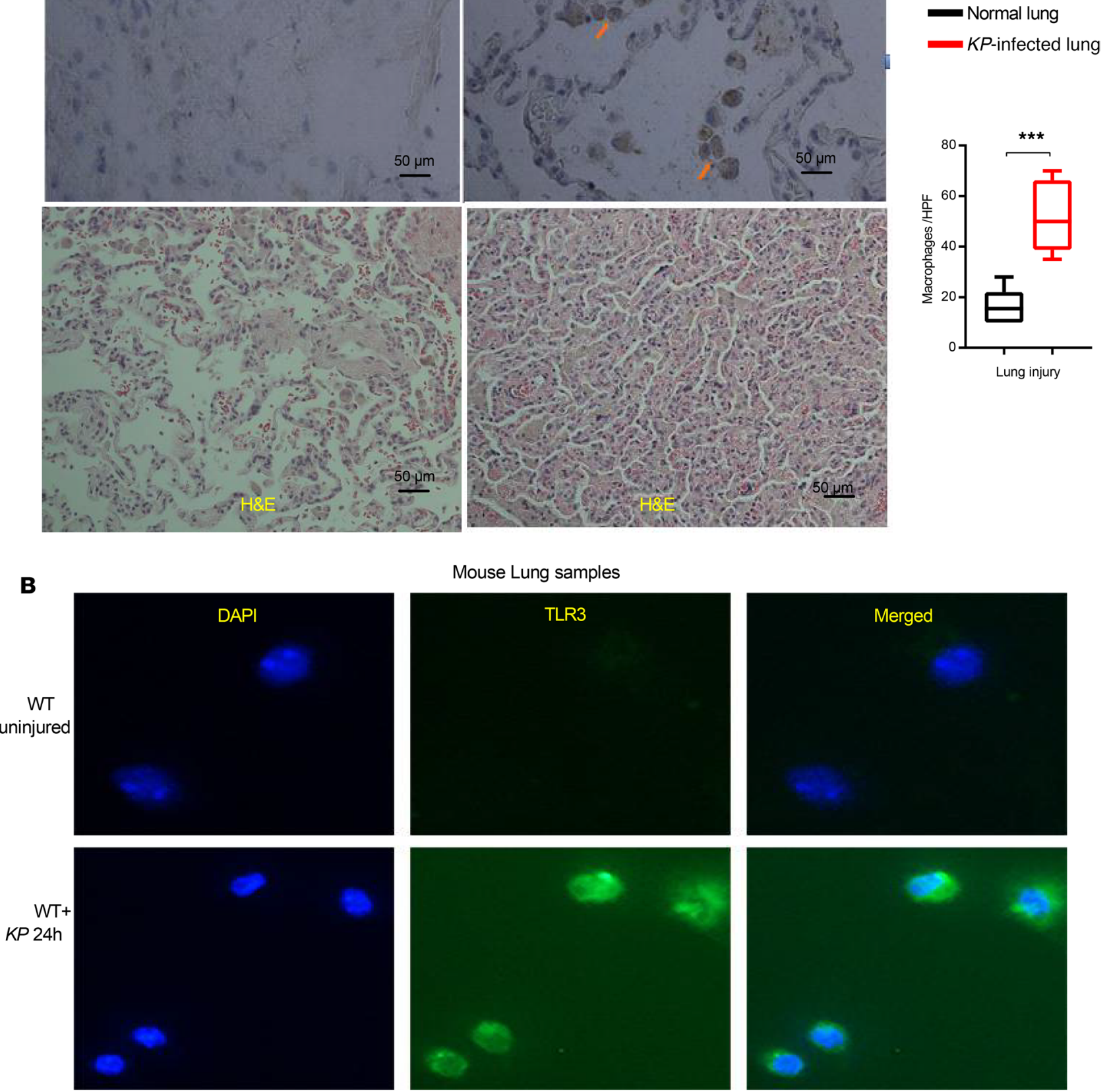

C

Capillary Immunoblotting

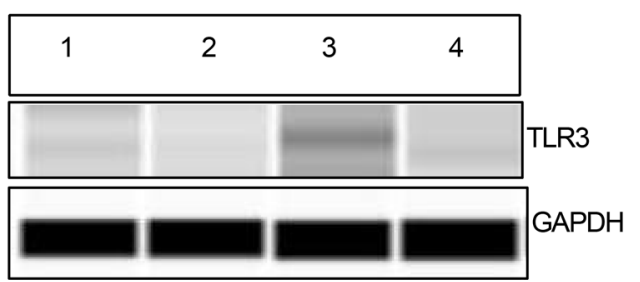

1. WT uninjured

2. TLR3-/- uninjured

3. $\mathrm{WT}+K P 24 \mathrm{~h}$

4. TLR3 -/-+KP $24 \mathrm{~h}$

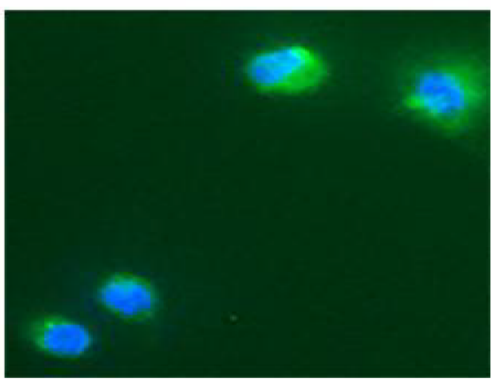


Figure 1. Postmortem lungs from patients with $K \boldsymbol{P}$ show significant expression of TLR3. (A) Postmortem lung samples in patients with $K P$ compared to normal lung tissue. Representative IHC images from a normal human lung stained with anti-TLR3 antibody and a lung with $K P$ stained with antiTLR3 antibody. Histopathological evaluation of postmortem lung samples ( $n=11 /$ samples) from controls and patients with $K P$ (***P $P 0.001$ human normal lung vs. KP-infected lung). Interestingly, intense expression of TLR3 was also observed in 2 patients with deaths attributed to Acinetobacter infection $(n=10)$ (Table 1). (B) Immunocytochemistry: TLR3 expression in isolated alveolar macrophages from WT mice at 24 hours following KP. Samples were subjected to staining with TLR3 antibody (green) and nuclear staining with DAPI (blue) ( $n=3 /$ group). Statistical analysis was performed at each time point. Samples were analyzed using 2 -tailed unpaired $t$ test with Welch's correction $\left({ }^{*} P<0.05\right.$ WT uninjured vs. WT injured). (C) Capillary Western blot. TLR3 protein expression was determined at 24 hours following KP inoculation. Lung samples from 4 groups of mice (WT, TLR3 ${ }^{--}$uninjured, WT + KP 24 hours, TLR3-1- KP 24 hours were homogenized and subsequently lysed in RIPA buffer. Following Western immunoassay (Wes) protocol from Protein Simple, samples were loaded onto a plate and then tested using polyclonal TLR3 antibody (PA5-29619, eBioscience) and HRP-conjugated secondary antibodies (1:10, Anti-Rabbit Secondary Antibody, 042-206, Protein Simple). Data were analyzed using Protein Simple software to display bands. The box plots depict the minimum and maximum values (whiskers), the upper and lower quartiles, and the median. The length of the box represents the interquartile range.

We examined BAL albumin levels using ELISA at 24 and 48 hours following $K P$ administration. BAL albumin levels (a marker of permeability injury) were highest following LC but were significantly decreased in $T L R 3^{-/-}$mice compared with WT (Figure 3A) following $K P$. Levels of IL-1 $\beta$ and macrophage inflammatory protein 2 (MIP-2) were significantly reduced in $T L R 3^{-1-}$ mice following primary $K P$ infection (Figure $3, \mathrm{~B}, \mathrm{C}$, and E). However, BAL levels of $\mathrm{KC}$, a neutrophil-recruiting chemokine, were higher in the $T L R 3^{-1-}$ mice following primary pneumonia (Figure 3D).

We then examined blood and lung bacterial counts (CFU) in WT and TLR $3^{-1-}$ mice following $K P$ infection. There was a significantly reduced lung bacterial burden in the $T L R 3^{-/-}$mice following pneumonia at the 24- and 48-hour time points (Figure $4 \mathrm{~A}$ ). $T L R 3^{3^{--}}$mice had evidence of very minimal to no bacteremia in the blood at 24- and 48-hour time points following $K P$ treatment compared with WT mice (Figure $4 \mathrm{~B}$ ). The bacterial burden in the spleen was significantly reduced in the $T L R 3^{-1-}$ mice (Supplemental Figure $1 \mathrm{~A}$; supplemental material available online with this article; https://doi.org/10.1172/jci.insight.131195DS1). Gross morphological analyses of the $T L R 3^{-/-}$mice revealed reductions in lung injury and inflammation following pneumonia (Figure 4C). Additionally, histological evaluation of lung sections demonstrated significantly worse injury in WT compared with $T L R 3^{--}$mice at all time points (Figure 4D). There was a large area of intra-alveolar hemorrhage, necrosis, and proteinaceous material in WT mice after $K P$ infection at 24 and 48 hours. None of these features was seen in the TLR $3^{-1-}$ mice. Taken together, the data suggest that the increased mortality in the WT group was likely the result of progressive pneumonia and sepsis with positive blood cultures.

\section{Lung injury and inflammation with LPS}

We next wanted to determine whether the improved outcomes in the $T L R 3^{-1-}$ mice were the result of better bacterial clearance or a reduced response to lung injury. Because LPS is a major component in gram-negative bacteria, including $K P$, and a virulent antigen that can induce pneumonia and acute lung injury (1719), we wanted to test outcomes in WT and $T L R 3^{-/-}$mice to a fixed dose of LPS. To determine the extent of lung injury, pressure-volume (PV) mechanics were measured at 24- and 48-hour time intervals following LPS administration. The PV measurements showed significantly decreased compliance (increased lung injury) at the 24-hour time points in the $T L R 3^{-/-}$mice compared with WT mice (Figure 5, A and B). BAL albumin levels (a marker of permeability injury) were not significantly different between $T L R 3^{-1-}$ and WT mice at either time point (Figure 5C). Interestingly, levels of IL-1 $\beta$ and MIP-2 were lower in TLR $3^{-/-}$mice (Figure 5, D and E), but KC levels were higher at 24 hours (Figure 5F). Finally, histopathological examination of $\mathrm{H} \& \mathrm{E}$-stained lung tissue and measurement of lung injury score revealed no significant differences in the extent of the microscopic injury and the inflammatory response (Figure 5G). Taken together, these results suggest that the reduction in lung injury and inflammation in the WT and $T L R 3^{-1-}$ mice following $K P$ infection is not related to a direct injury on the parenchyma but rather is a result of improved bacterial clearance in the $T L R 3^{-/-}$mice.

\section{dsRNA released from necrotic cells is the ligand for activation of TLR3 in KP}

$T L R 3^{-1-}$ mice show similar intracellular staining for dsRNA but different extracellular staining following lung contusion and pneumonia. We next examined the expression of dsRNA following pneumonia. Intracellular staining of TLR3 and flow cytometric analysis revealed significant upregulation of TLR3 in WT mice infected with $K P$, which was ameliorated in the TLR3-knockout mice even though $K P$ induced similar levels of dsRNA in lung homogenates in both strains (Figure 6A). 
Table 1. IHC data show cytoplasmic and/or nuclear staining defining TLR3+ cells

\begin{tabular}{|c|c|c|c|c|c|c|c|c|}
\hline Serial number & Age/Sex & Blood & Trachea & BAL & ICU LOS & Grading & IHC scoring & $\begin{array}{c}\text { No. of positive } \\
\text { cells/HPF }\end{array}$ \\
\hline 1 & $55 / M$ & Sterile & $\begin{array}{c}\text { Klebsiella } \\
\text { pneumoniae }\end{array}$ & No & 9 & 3 & 3 & 2-10/HPF \\
\hline 2 & $46 / M$ & GNB & $\begin{array}{c}\text { Acinetobacter } \\
\text { baumanii }\end{array}$ & No & 12 & 1 & 0 & 0 \\
\hline 3 & $27 / M$ & GNB & No & GN/GP & 9 & 2 & 2 & 2-5/HPF \\
\hline 4 & $62 / M$ & $\begin{array}{c}\text { Pseudomonas } \\
\text { aeruginosa }\end{array}$ & GNB - multiple & Upper respiratory & 63 & 3 & 3 & 2-10/HPF \\
\hline 5 & $46 / M$ & No & $\begin{array}{c}\text { Klebsiella } \\
\text { pneumoniae }\end{array}$ & $\begin{array}{c}\text { Acinetobacter } \\
\text { baumanii }\end{array}$ & 7 & 2 & 1 & 1/20HPF \\
\hline 6 & $34 / M$ & Sterile & $\begin{array}{c}\text { Acinetobacter } \\
\text { baumanii }\end{array}$ & No & 14 & 0 & 2 & 1-2/10HPF \\
\hline 7 & $65 / M$ & $\begin{array}{c}\text { Acinetobacter } \\
\text { baumanii }\end{array}$ & No & No & 4 & 0 & 1 & 1-2/10HPF \\
\hline 8 & $23 / F$ & Sterile & $\begin{array}{c}\text { Acinetobacter } \\
\text { baumanii }\end{array}$ & No & 36 & 2 & 2 & 1-2/10HPF \\
\hline 9 & $44 / M$ & Sterile & No & $\begin{array}{c}\text { Acinetobacter } \\
\text { baumanii }\end{array}$ & 12 & 3 & 3 & 1-2/10HPF \\
\hline 10 & $41 / M$ & GN & No & No & 24 & 2 & 3 & 5-10/HPF \\
\hline \multicolumn{9}{|c|}{$\begin{array}{l}\text { The blood and tracheal cultures of patients infected with Pseudomonas and Acinetobacter infections show a higher expression of TLR3. For IHC, cells with } \\
\text { cytoplasmic and nuclear staining found TLR3+ cells. For IHC, cells with cytoplasmic and/or nuclear staining were defined as TLR3 }{ }^{+} \text {cells. BAL, bronchoalveola } \\
\text { lavage; ICU LOS, intensive care unit length of stay; HPF, high-power field; GNB, gram-negative bacteria; GN, gram-negative; GP, gram-positive. }\end{array}$} \\
\hline
\end{tabular}

We next examined the relationship between dsRNA and TLR3 in the whole-lung lysate. With experimental repeats (3 times), we co-immunoprecipitated dsRNA antibody in the lysate and then blotted for TLR3. We found that dsRNA was present even in uninfected alveolar macrophages, but levels of TLR3 were not substantially increased till 48 hours after $K P$ (Figure $6 \mathrm{~B}$ ). The kinetics of these experiments suggested to us that it was not the amount of dsRNA that drives the differential degree of inflammatory response between the $\mathrm{KO}$ and WT mice. It is the amplification of the inflammatory response in the WT that results in increased permeability injury (BAL albumin) and inflammation (cytokines).

$T L R 3 / d s R N A$ complex inhibitor improves mortality in WT mice following KP. WT mice were administered TLR3/dsRNA complex inhibitor 1 hour before bacterial infection, which resulted in significantly reduced mortality following bacterial pneumonia (Figure 6C). These results suggest that injury with bacterial pneumonia and LC increases the amount of dsRNA, generating an amplified inflammatory response in the presence of TLR3.

\section{A central role for alveolar macrophages in TLR3-driven pathology following $K P$}

Alveolar macrophages from TLR $3^{-1-}$ mice have substantially improved bactericidal and phagocytic activity compared with $W T$. It has been previously reported that depletion of alveolar macrophages results in increased mortality with $K P(6)$. Previous studies also showed that the capsule of $K P$ reduces phagocytosis by alveolar macrophages $(20,21)$. Using a phagocytic assay, we evaluated isolated alveolar macrophages from WT and TLR $3^{-1}$ uninjured and injured ( $K P$-infected) mice that were treated with FITC-labeled $K P$. Both the serum-opsonized and nonopsonized relative phagocytic activity was significantly increased at 24 hours in $T L R 3^{-1-}$ mice compared with WT mice (Figure 7A). Next, we performed single-cell imaging of alveolar macrophages isolated in the presence and absence of FITC-labeled dead $K P$ from both genotypes. There were significant differences in phagocytic activity in $T L R 3^{-/-}$mice compared with WT mice (Figure $7 \mathrm{~B}$ ), with $T L R 3^{-/-}$alveolar macrophages showing enhanced uptake of FITC-labeled heat-killed bacteria. Additionally, we examined the phagocytosis of live $K P$. Macrophages were harvested from WT and $T L R 3^{--}$mice at 24 hours following live $K P$ administration and subjected to immunofluorescence staining with anti-Klebsiella antibody (shown in green) and nuclear staining with DAPI (blue). The fluorescent images show intense phagocytosis in the TLR3 ${ }^{-/-}$macrophages following $K P$ administration compared with WT mice (Figure 7C). These results suggest that in the absence of TLR3, macrophage phagocytic capacity is enhanced, which in turn confers a protective effect from pneumonia infection while reducing the mortality rate resulting from the acute inflammatory response. 
Table 2. Pneumonitis grading score

\begin{tabular}{|c|c|}
\hline Grade & Histopathological findings \\
\hline 0 & None \\
\hline 1 & Alveolar thickening $20 \%$; alveolar spaces normal \\
\hline 2 & Alveolar septal thickening $20 \%-50 \%$ \\
\hline 3 & Alveolar septal thickening $>50 \%$; consolidation with mononuclear infiltrates in the alveolar space \\
\hline
\end{tabular}

Moreover, we determined the bactericidal activity in isolated alveolar macrophages from both naive and $K P$-administered mice. Alveolar macrophages from $T L R 3^{-1-}$ mice were better able to kill both opsonized and nonopsonized bacteria (Figure 7D). Finally, the evaluation of Klebsiella hemolysin gene (Khe) expression in the surviving $K P$ population within the alveolar macrophages confirmed these findings (Figure 7E). Taken together, these data suggest that the alveolar macrophages in $T L R 3^{-1-}$ mice drive bacterial clearance through the enhanced bactericidal activity as well as increased phagocytic capability.

The role for alveolar macrophage efferocytosis following pneumonia. Efferocytosis (the phagocytosis of apoptotic self-cells) is a key mechanism allowing engulfment by phagocytes of dying and dead cells as well as their debris, and this process is important in the resolution of various pathologies $(22,23)$. To investigate this process, we first used the mouse macrophage ( $\mathrm{MH}-\mathrm{S}$ cell line) co-incubated with apoptotic cells (camptothecin-treated human Jurkat T cell line). The results demonstrated that macrophage cells co-incubated with apoptotic cells showed efficient efferocytosis (Figure 8A). We next isolated alveolar macrophages from WT and $T L R 3^{-/-}$mice that were uninfected or infected with $K P$ for 24 hours and tested the efferocytotic capacity of these cells ex vivo. Here we found that efferocytosis was increased in macrophages originating from the $T L R 3^{-1-}$ mice compared with WT mice in both the uninjured and injured groups (Figure 8B), but this was significant only after 24 hours.

The alveolar macrophage phenotype polarization following pneumonia. Macrophage polarization into M1 or M2 phenotype dictates the nature, duration, and severity of an inflammatory response (4, 24, 25). WT and $\mathrm{TLR}^{-/-}$mice were subjected to $K P$, and RNA was isolated from the BAL macrophages. Levels of NOS-2, arginase-1, FIZZ-1, and IL-10 genes were measured by a quantitative reverse transcriptase PCR. The expression of NOS-2 was significantly reduced at the 24-hour time point in the $T L R 3^{-/-}$mice compared with WT mice (Figure 9A). On the other hand, the levels of arginase-1, FIZZ-1, and IL-10 were significantly higher in $T L R 3^{-/-}$mice at 24 hours compared with WT mice (Figure 9, B-D). Correspondingly, the $T L R 3^{-1-}$ mice exhibited a protective M2 phenotype as evidenced by increased arginase-1 and FIZZ-1 expression. Taken together, these data suggest TLR3 deletion promotes skewing to predominant M2 polarization in alveolar macrophages characterized by counterinflammatory gene products, while maintaining a high phagocytic capability for both $K P$ and apoptotic cells.

\section{Table 3. IHC score}

$\begin{array}{cc}\text { Score } & \text { Intensity } \\ 0 & \text { Nil } \\ 1 & \text { Mild } \\ 2 & \text { Strong }\end{array}$

Statistical analysis was used and scores of IHC = scores of staining intensity $\times$ scores of the percentage of positive cells ( $\tau_{b}=0.6571$, and $P=0.03$ ). The percentage of positive cells was scored as: $0=0 \%-5 \%, 1=6 \%-25 \%, 2=26 \%-50 \%$, and $3=51 \%-100 \%$. 
A
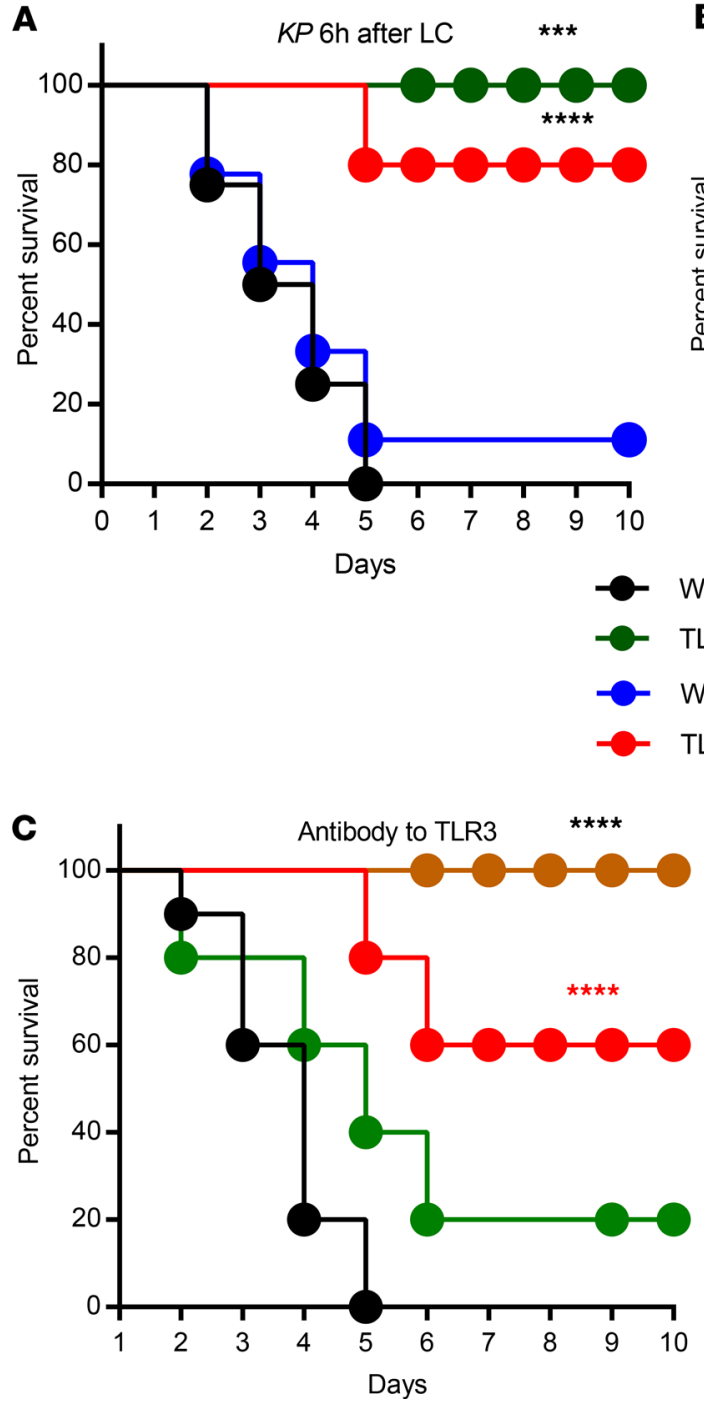

B $\quad K$ concurrently after $L C$

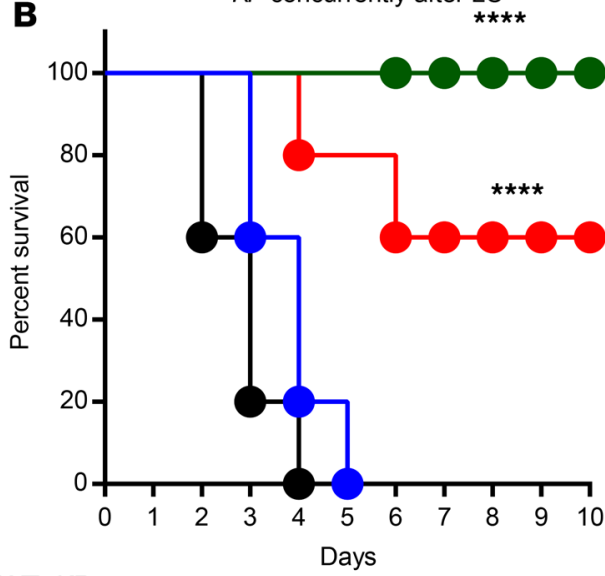

$W T+K P$

TLR3(-/-)+KP

WT LC+KP

TLR3(-/-) LC+KP

Figure 2. TLR3 controls host defense against $K P$ infection: survival study following $K P$. WT and $T L R 3^{-/-}$mice were administered (via pharyngeal drops) with 500 CFU of KP 6 hours following (A) or concurrently with LC (B), and mortality was monitored up to 10 days ( ${ }^{*} P<0.05$ by log-rank test). Data shown represent $n=20$ mice/group from 4 independent experiments ${ }^{* * *} P<0.001$, and ${ }^{* * * *} P<0.0001$, WT vs. TLR3 $3^{-1-}$. TLR3 antibody neutralization survival study: administration of TLR3 antibody (50 and $100 \mu \mathrm{g})$ protected the WT mice following $K P$ (C) $\left(n=10 /\right.$ group) ${ }^{* * * *} P<0.0001$ by log-rank test, WT vs. TLR3 antibody-administered WT mice).

Enhanced chemokine secretion from TLR $3^{--/}$mice. We conducted RNA-sequencing analysis to elucidate the role of TLR3 following pneumonia. RNA-sequencing analysis of alveolar macrophages revealed that the TLR3-1mice produced significantly higher amounts of chemokines, particularly, CXCL1 (KC) and -2 (MIP-2). TLR3 activation results in diminished chemokine production of CXCL1, -2, and -10 by alveolar macrophages (Figure $9 \mathrm{E})$. These data suggest neutrophilic chemoattractants play a functionally important role in the promotion of host defense in bacterial infections by attracting neutrophils that play a key role in bactericidal activity.

Decreased macrophage apoptosis in TLR $3^{-1-}$ mice. We next assessed the degree of lung injury and the inflammatory profile by comparing apoptotic responses in WT and TLR $3^{--}$mice. We examined the responses based on alveolar macrophage phenotype polarization in WT and $T L R 3^{3^{-1}}$ mice following primary pneumonia. M1 macrophage apoptosis was significantly lower following primary pneumonia at 24 hours, but both M1 and M2 macrophage apoptosis were significantly lower in TLR $3^{---}$mice following 24 hours' pneumonia compared with WT mice (Figure 10, A and B). These data suggest $T L R 3^{---}$alveolar macrophages are better able to resist apoptosis regardless of the polarization state. 

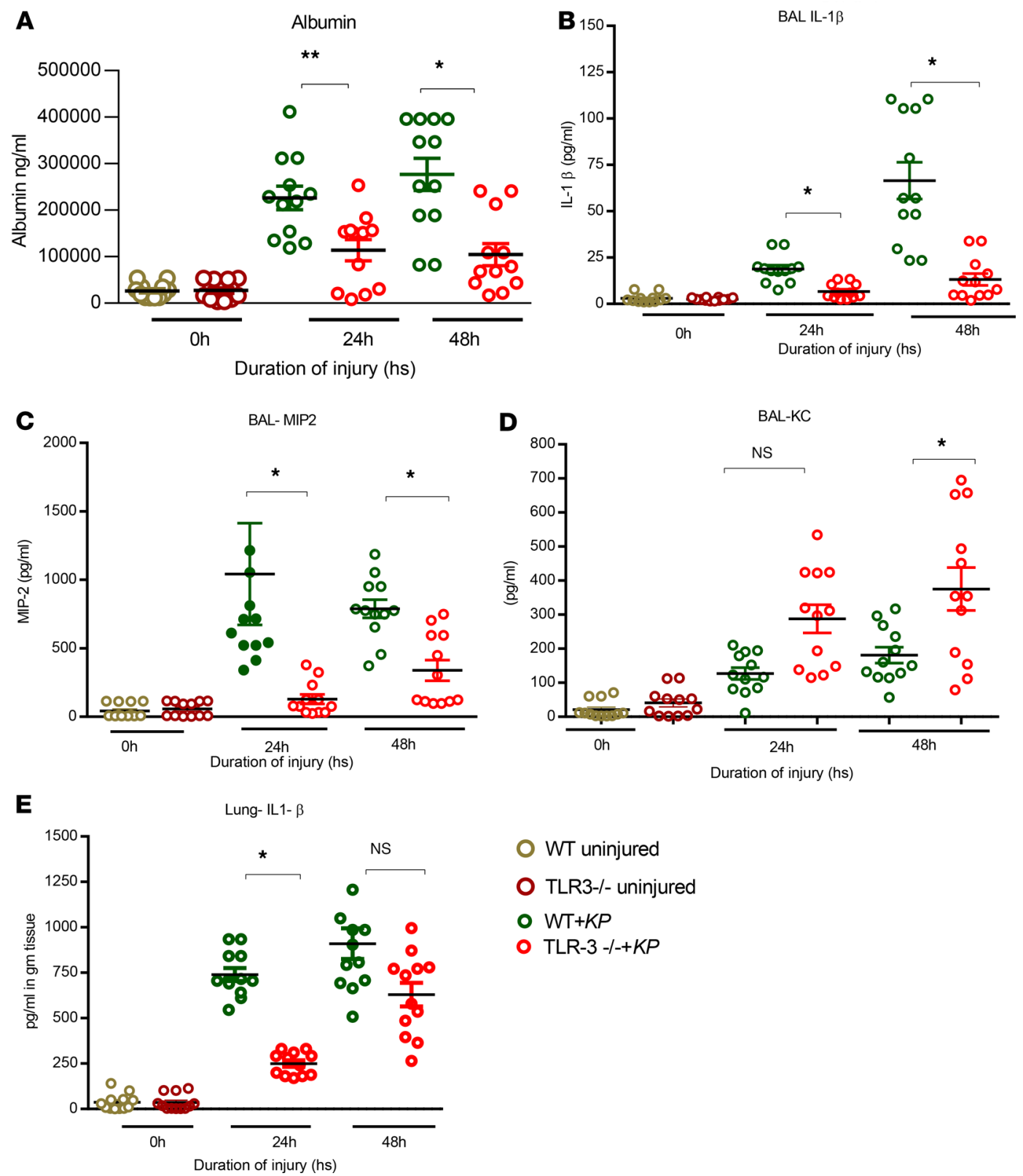

Figure 3. WT mice show increased lung injury and inflammation compared with TLR3 ${ }^{-1-}$. WT mice had increased albumin levels following primary

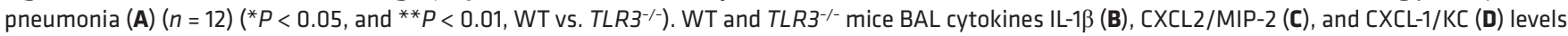
following primary pneumonia ( $n=12 /$ group) measured by ELISA. The levels of lung cytokine IL-1 $\beta$ (E) were measured by using ELISA ( $n=12 / \mathrm{group}$ from 3 independent experiments). Statistical analysis was performed at each time point. Samples were analyzed using 2-tailed unpaired $t$ test with Welch's correction $\left({ }^{*} P<0.05\right.$ WT vs. $T L R 3^{-/-}$mice $)$.

Role of neutrophils following pneumonia

Increased neutrophils in TLR $3^{--}$mice. Neutrophils are the first phagocytic cells recruited to the site of bacterial infection. Numerous reports suggest that TLR 3 is predominantly located on alveolar macrophages and is not present on peripheral circulating neutrophils $(26,27)$. Here, we examined the levels of neutrophils in BAL fluid and lungs collected from WT and $T L R 3^{-/-}$mice at different time intervals following $K P$. The data show no significant difference in neutrophil levels in the airspaces between WT and $T L R 3^{-1-}$ mice at any time (Figure 11A). However, following $K P$ inoculation there was a significantly higher level of neutrophils in the $T L R 3^{-/-}$mice lungs at the 24-hour time point compared with WT mice (Figure 11B). We next tested for mediators of neutrophil functional activity, such as NO (28), which is known to be important in host defense against pathogens $(29,30)$. We found no significant NO difference in the BAL between WT and 
A

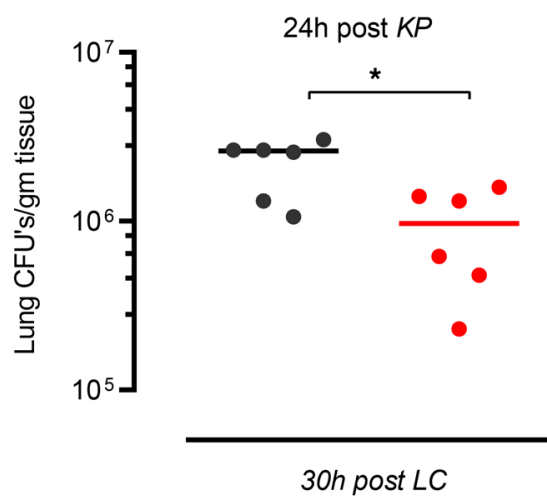

B

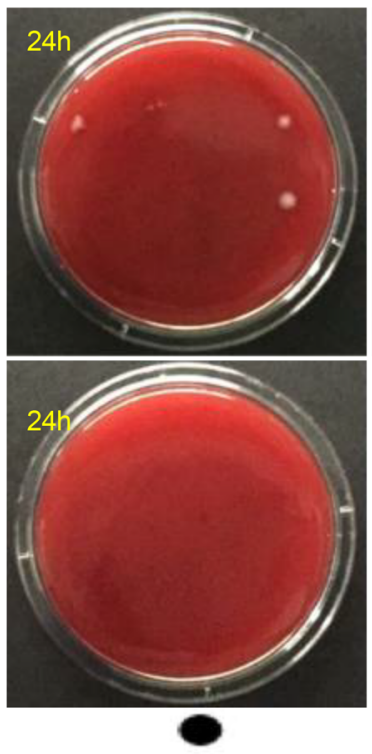

Blood culture

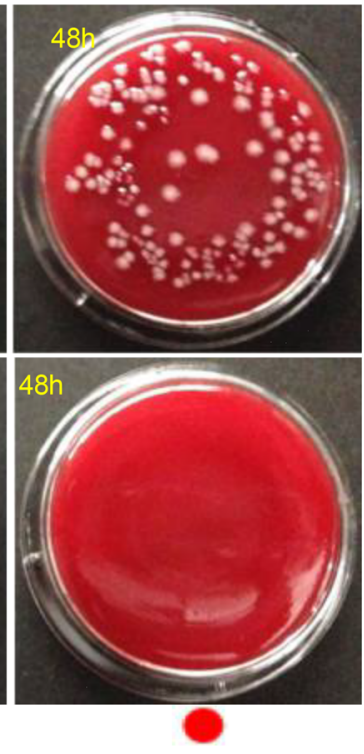

Lung culture

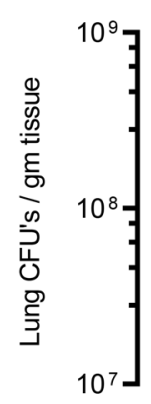

48h post $K P$

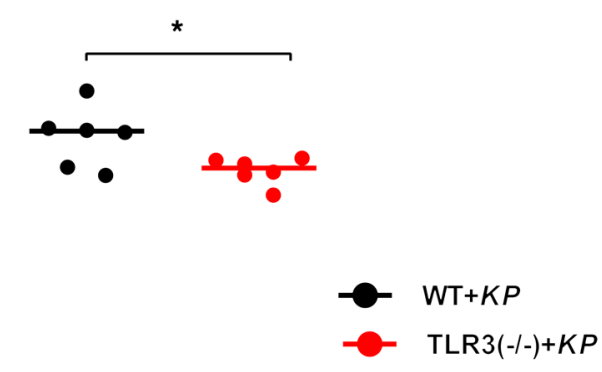

54h post LC

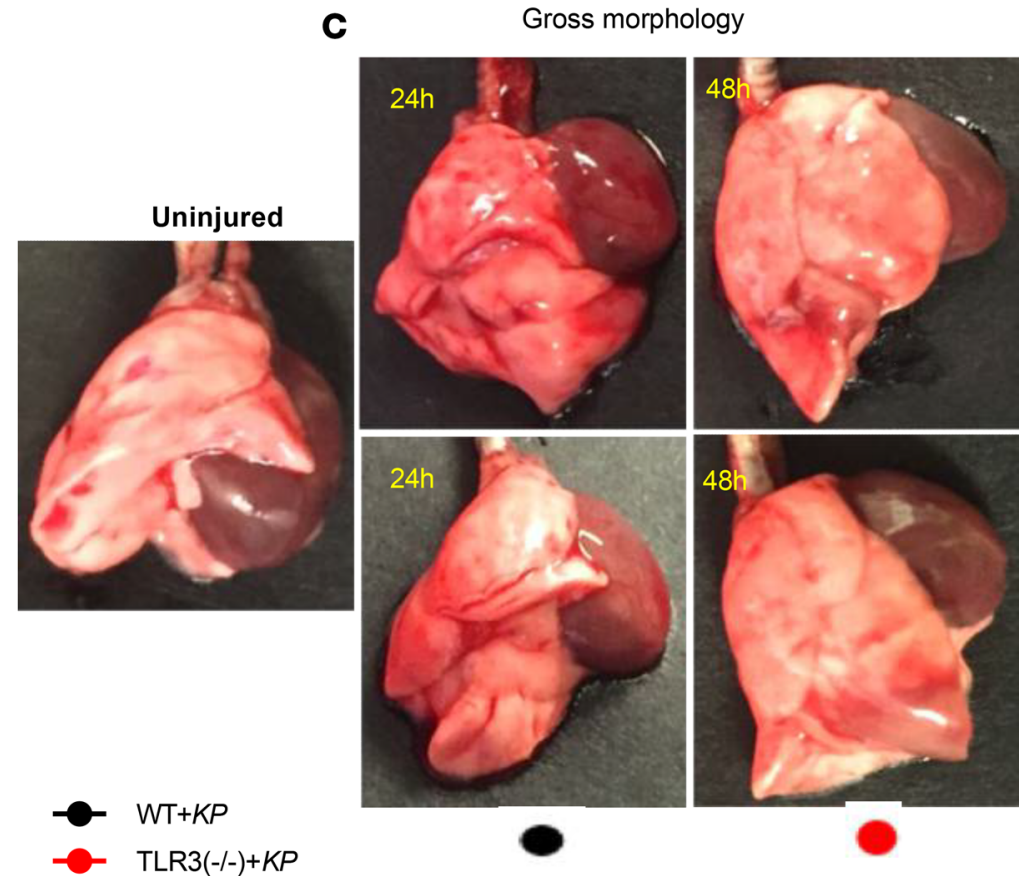

\section{D}

$24 \mathrm{~h}$
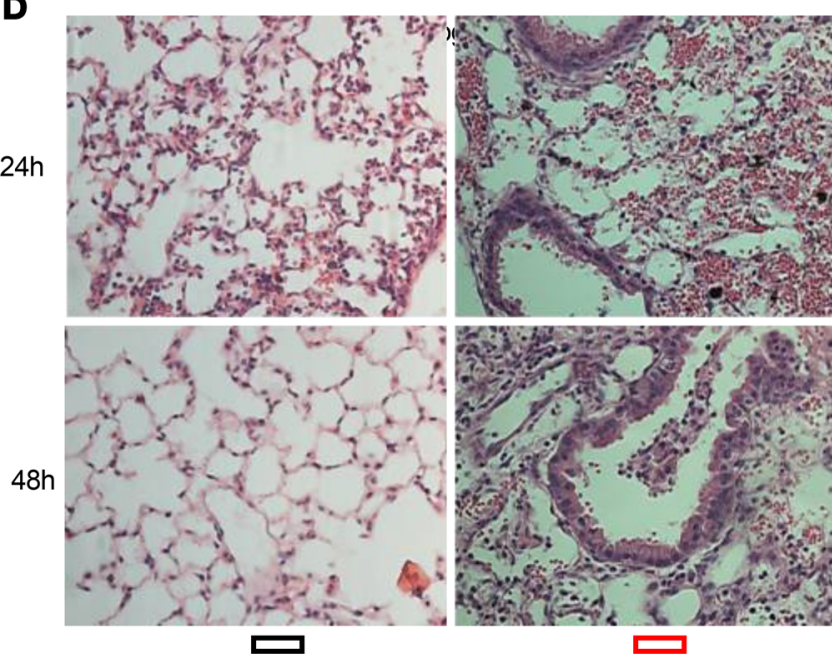

Pathological score

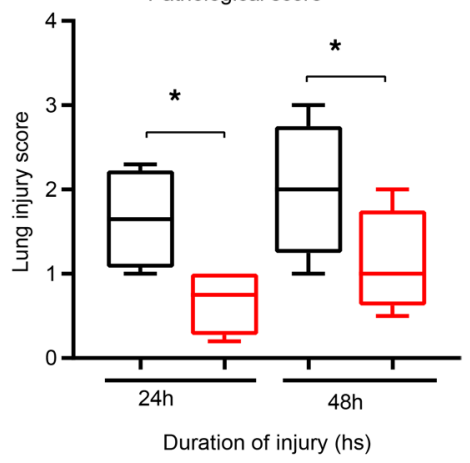

WT $+K P$
TLR3-/-+KP 
Figure 4. Reduced bacteremia and gross morphology following pneumonia administration. $T L R 3^{-1-}$ mice had reduced lung bacterial CFU (A) in blood culture (bacteria growth) (B) and whole lung gross morphology (C) at 24 and 48 hours compared with WT mice ( $n=6$ mice per group, 2 independent experiments). Left side (black bullet) is WT+KP, and right side (red bullet) is $T L R 3^{-1-}+K P$. Gross morphological analyses of the $T L R 3^{-1-}$ mice revealed reductions in lung inflammation following $K P(n=6$ mice per group, 2 independent experiments) (D) Histology: WT and TLR3 mice were inoculated with 500 CFU of $K P$ per mouse. Lungs were collected at 24 and 48 hours after infection and stained with $\mathrm{H} \& \mathrm{E}$, and inflammatory changes in histological sections were scored. Shown are representative sections from 3 mice per each condition (original magnification, $\times 200$ ). Statistical analysis was performed at each time point. Samples were analyzed using 2-tailed unpaired $t$ test with Welch's correction ( ${ }^{*} P<0.05$ WT vs. TLR3 ${ }^{-1-}$ mice). The box plots depict the minimum and maximum values (whiskers), the upper and lower quartiles, and the median. The length of the box represents the interquartile range.

$T L R 3^{-1-}$ mice at all time points (Figure 11C). However, there was a significantly lower level of NO in the $T L R 3^{-1-}$ lung tissue at the 24-hour time point compared with WT mice following KP (Figure 11D). We next examined malondialdehyde (MDA) following pneumonia. MDA is a frequently used biomarker for oxidative stress (31). Using ELISA, we determined the levels of MDA in BAL fluid and in lung tissue collected from WT and $T L R 3^{-1-}$ mice at different time intervals following KP. The levels of BAL MDA were significantly lower in $T L R 3^{-1-}$ mice at the 48-hour time point compared with WT mice following $K P$ (Figure $11 \mathrm{E}$ ).

Neutrophil depletion. We next sought to elucidate the relationship between TLR3 knockout and neutrophil activity and impact on survival following $K P$. WT and $T L R 3^{-1-}$ mice received anti-Ly6G antibody via intraperitoneal injection to deplete neutrophils. After 48 hours, blood was collected, and the hematological parameters were examined in the mice (Figure 12A). Neutrophil numbers were significantly reduced in both WT and $T L R 3^{-1-}$ mice that received anti-Ly6G antibody (intraperitoneally at $170 \mu \mathrm{g}$ per mouse in $200 \mu \mathrm{L}$ ) compared with the untreated mice (Figure 12A) following $K P$. These results showed the efficacy of this antibody treatment.

Next, we administered 500 CFU KP to both groups treated with Ly6G antibody and a separate group of WT and $T L R 3^{-1-}$ mice not given the antibody and monitored their survival. Additionally, 48 hours after $K P$, blood was collected, and the hematological parameters were examined in the mice. The number of neutrophils significantly increased in the injured WT mice that did not receive the antibody. However, the antibody effectively reduced neutrophils in both genotypes (Figure 12B). In our survival study, the neutrophil-depleted WT and $T L R 3^{-1-}$ mice both died in similar patterns as the WT mice with $K P$ alone (Figure 12C). Taken together, these data suggest neutrophils play a critical role in the survival of the $T L R 3^{-/-}$mice through an indirect effect.

Neutrophil contribution from the spleen. To evaluate whether the splenic reservoirs contributed to the neutrophils, we evaluated splenic neutrophils with histology and myeloperoxidase (MPO) measurements of the spleen. There were no significant differences in the number of neutrophils and amount of MPO between WT and $T L R 3^{--}$mice following KP infection at 24- and 48-hour time points (Supplemental Figure 1C). These results suggest that splenic contribution to the increased neutrophils was at best limited (Supplemental Figure 1C). We did not perform the definitive experiment involving splenectomy.

\section{Adoptive transfer}

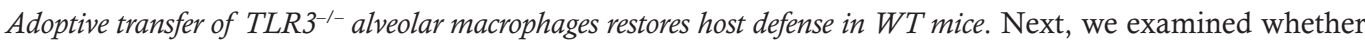
adoptive transfer of macrophages can rescue the WT mice following pneumonia infection. We isolated alveolar macrophages from $T L R 3^{-/-}$uninjured mice. After confirmation to establish the suspension contained more than $98 \%$ macrophages, the $T L R 3^{-/-}$macrophages were transferred to WT mice. $K P$ was administered 48 hours later to both groups, and survival was monitored for 10 days. Our results demonstrated that WT mice were rescued (70\%) following the introduction of $T L R 3^{-1-}$ macrophages compared with $100 \%$ mortality within 5 days in WT mice that received bacteria alone (Figure 13A). These data suggest the ability of alveolar macrophages from $T L R 3^{-/-}$mice to rescue the WT animal from $K P$ infection.

Adoptive transfer of $T L R 3^{-1-}$ alveolar macrophages improves bacterial clearance in WT mice. Additionally, in a separate experiment, we isolated alveolar macrophages from $T L R 3^{-1-}$ uninjured mice and then transferred them to WT mice. Pneumonia was administered 48 hours later to both groups, regardless of the presence or absence of $T L R 3^{-/-}$macrophages. Blood and lung cultures were conducted 24 and 48 hours following pneumonia infection. The blood culture data indicate a significant reduction at the 24-hour time point in blood culture between the WT mice given $K P$ alone and WT mice given $T L R 3^{-1-}$ macrophages and $K P$ (Figure $13 \mathrm{~B})$. There was a significant reduction in bacterial colonies in lung culture at both the 24-hour and 48-hour time points when comparing the same 2 groups (Figure 13C). In summary, we have provided conclusive evidence to show that the protective effect of the deletion of TLR3 in combating KP infection is mediated through the activity of the alveolar macrophages. 
A

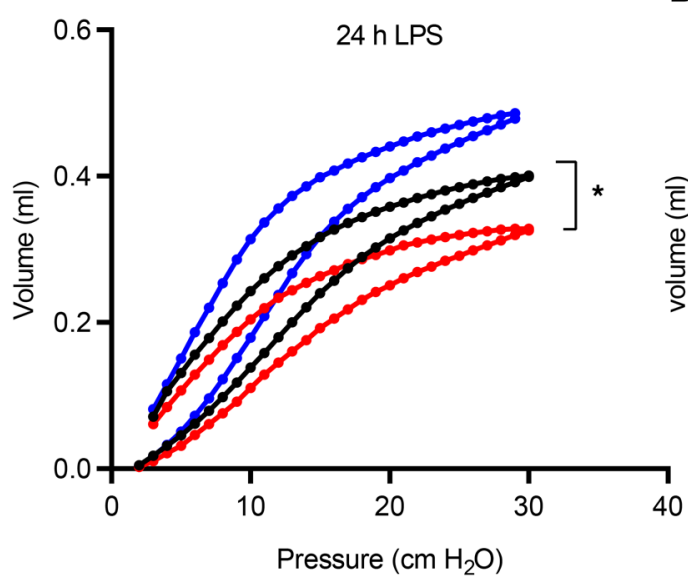

B

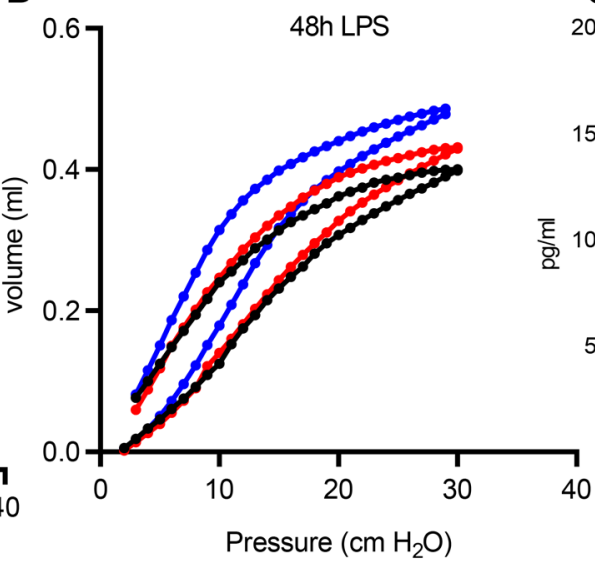

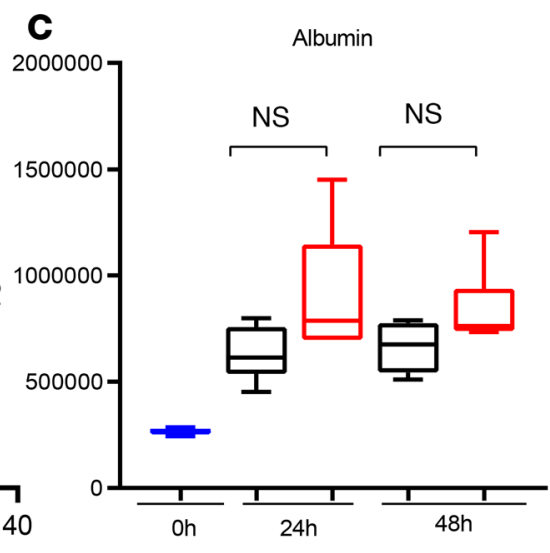

D
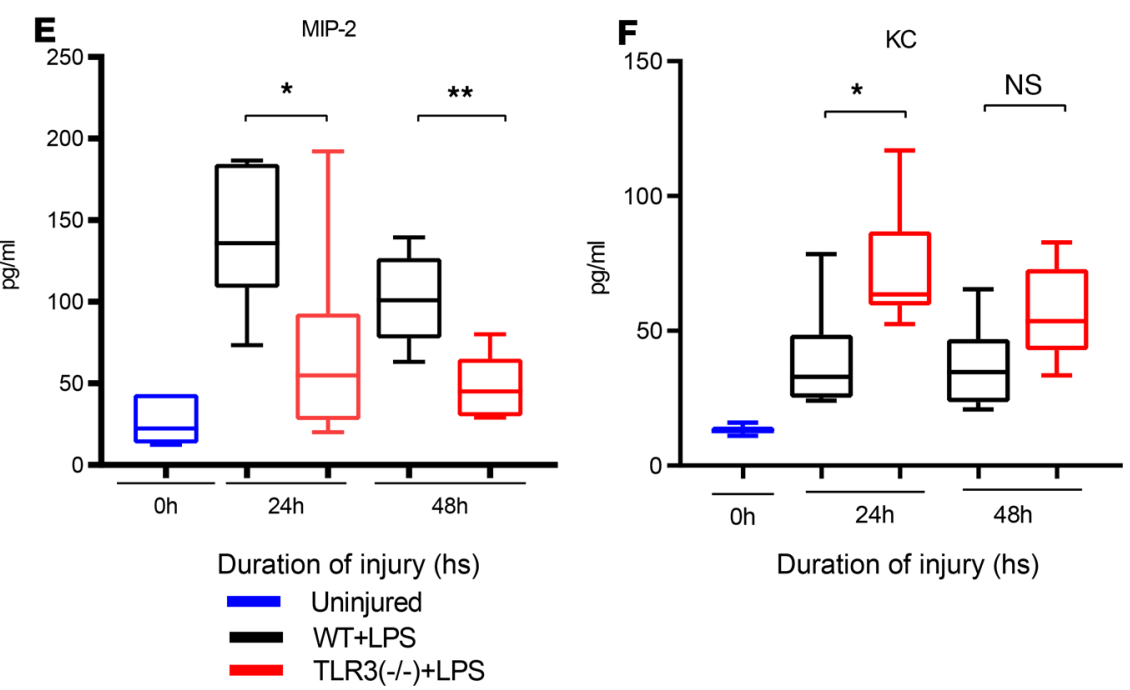

Duration of injury (hs)

G
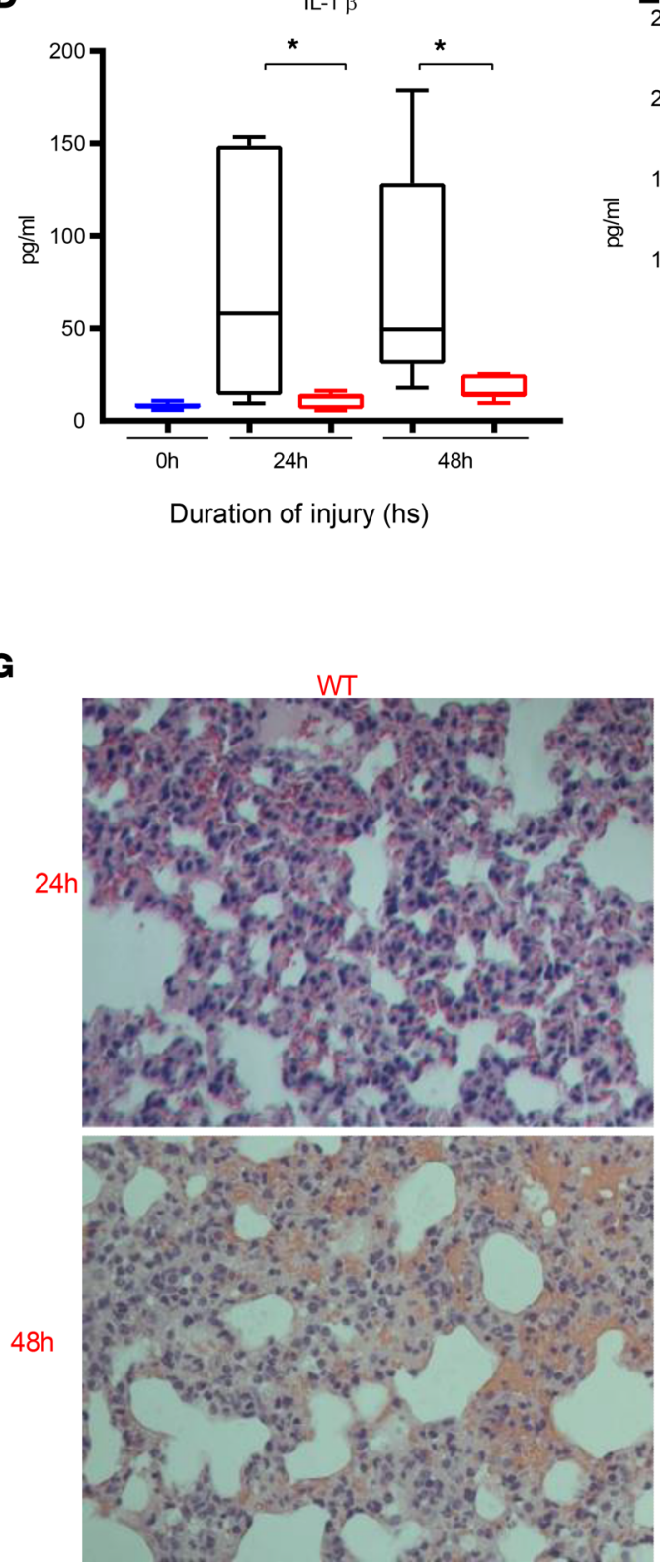

$\operatorname{TLR} 3(-/-)$
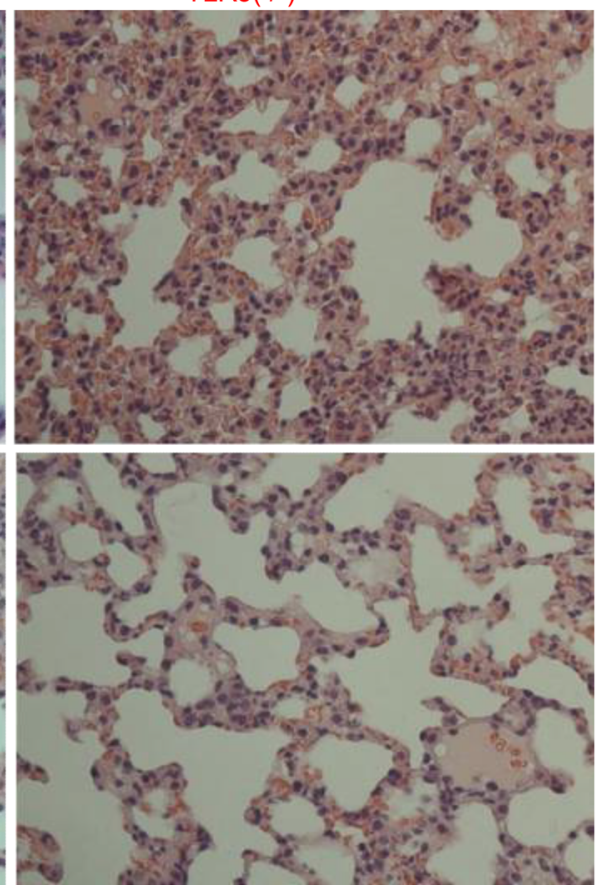

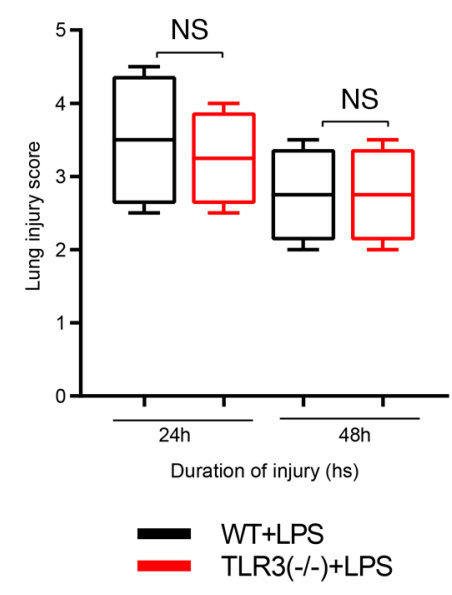


Figure 5. WT mice show increased inflammation following LPS administration. PV curves after established combined injury with LPS. PV curves at 24 hours (A) and PV curves at 48 hours (B). Using a SCIREQ FlexiVent quasistatic PV curves with maximal inflation to $30 \mathrm{cmH}_{2} \mathrm{O}$ were generated from over 4000 data points ( $n=6$ per group, 2 independent experiments). The bottom half of each loop represents the inspiratory portion of a PV curve perturbation maneuver. Top half represents the expiratory portion of each graph ( ${ }^{*} P<0.05$ WT vs. TLR3 $3^{-/-}$mice). (C) Mouse albumin was measured by ELISA at 24 and

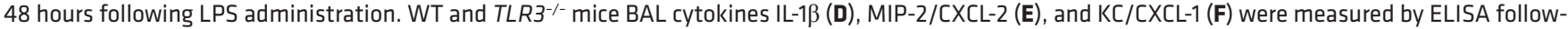
ing LPS ( $n=6$ per group, 2 independent experiments) $\left({ }^{*} P<0.05\right.$, and ${ }^{* *} P<0.01$, WT vs. TLR3 ${ }^{-1-}$ mice). (C) WT and TLR3 mice were inoculated with LPS, and lungs were collected at 24- and 48-hour time points and stained with H\&E. Shown are representative sections ( $n=3$ per group) from each condition (original magnification, $\times 200$ ). Statistical analysis was performed at each time point. Samples were analyzed using 2-tailed unpaired $t$ test with Welch's correction ( ${ }^{*} P<0.05$ WT vs. TLR3 $3^{-1-}$ mice). The box plots depict the minimum and maximum values (whiskers), the upper and lower quartiles, and the median. The length of the box represents the interquartile range.

Adoptive transfer of TLR $3^{-/-}$alveolar macrophages increases neutrophil activity in WT mice. Neutrophils have crucial antimicrobial functions but are also thought to contribute to tissue injury upon exposure to bacterial products (32). Therefore, an important step in the initiation of an immune response is the synthesis of the neutrophil-recruiting chemokines. Previous studies described that peripheral neutrophils do not express TLR3 $(26,27)$. Here, we examined neutrophil activity following adoptive transfer. The mice lung lysate was collected, and the levels of keratinocyte chemoattractant (KC) and MIP-2 were measured by ELISA. The levels of KC and MIP-2 were significantly higher in the mice that received $T L R 3^{-1-}$ alveolar macrophages in combination with $K P$ compared with $K P$ alone group (Figure 14, A and B). Finally, histological evaluation of $\mathrm{H} \& \mathrm{E}$-stained lung sections revealed that the numbers of neutrophils and macrophages were significantly increased in the $T L R 3^{-/-}$alveolar macrophage adoptive transferred mice compared with the WT mice that received $K P$ alone (Figure 14, C and D). Taken together, these data suggest that adoptive transfer of $T L R 3^{-/-}$alveolar macrophages induces the production of chemokines (KC and MIP-2) and recruitment of neutrophils constituting a first essential step in response to a pathogen.

\section{Discussion}

Bacterial pneumonia, particularly in the setting of the VAP, is the leading cause of nosocomial infections. The incidence of VAP continues to be steady despite the introduction of the VAP bundle in the care of critically ill patients. LC injury associated with barotrauma is the most common critical injury sustained by people during blast damage in both civilian and military settings (33). LC is also an independent risk factor for the development of acute respiratory distress syndrome (34) and VAP in affected patients (35). Gram-negative organisms are typically responsible for $70 \%$ of the VAP incidences. We have specifically chosen to study $K$. pneumoniae because it accounts for a significant proportion of community- and hospital-acquired pneumonia. $K P$ is particularly virulent because it causes significant alveolar necrosis and has the potential to cause septicemia. We have recently demonstrated that TLR3 plays a major role in the initiation and maintenance of acute inflammatory response following LC (4). This study was undertaken to explore the effects of TLR3 on $K P$ alone and $K P$ after LC.

TLR3 has been considered a pathogen recognition molecule associated with viral infections, with dsRNA serving as its sole ligand. TLR3 activation stimulates production of type I IFN as the main mechanism for generating antiviral immunity. Following blunt trauma-induced LC, TLR3 acts as a sensor of necrotic cells and drives the majority of inflammation following LC (4) and other acute inflammatory events (12). Here we observed a finding related to gram-negative bacterial pneumonia. Although all WT mice following inoculation with $K P$ died by day 5, 19 of the $20 T L R 3^{-/-}$mice survived the insult. All the $T L R 3^{-/-}$mice with primary $K P$ infection survived. The survival pattern was replicated in the setting of secondary bacterial pneumonia, where the mice received LC followed by inoculation with $K P$ administered either concurrently or 6 hours following LC. A similar survival pattern was seen with the monoclonal antibody neutralization, confirming the effect was TLR3 specific.

An additional key observation was the presence of reduced permeability lung injury and inflammation in the $T L R 3^{-1-}$ mice. The degree of inflammation and the nature of the cytokine response indicates that the acute and subacute inflammation in the WT compared with $T L R 3^{-1-}$ mice persist over a period. However, there is clear evidence that the bacterial burden in the WT mice at any time point is higher than the KO mice. To eliminate the confounding variable of the increased bacterial burden, we used LPS to induce lung injury and inflammation. LPS has been widely reported to interact with TLR3 directly (36). As shown in Figures 3 and 4, we did not see any significant differences in permeability (BAL albumin) or inflammation as seen by histology and cytokine levels. Therefore the increased survival seen in $T L R 3^{-/-}$mice is primarily due to improved bacterial clearance and not a result of reduced lung injury. 


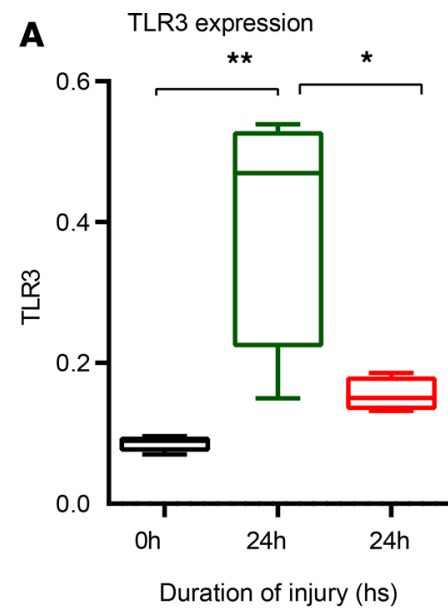

B

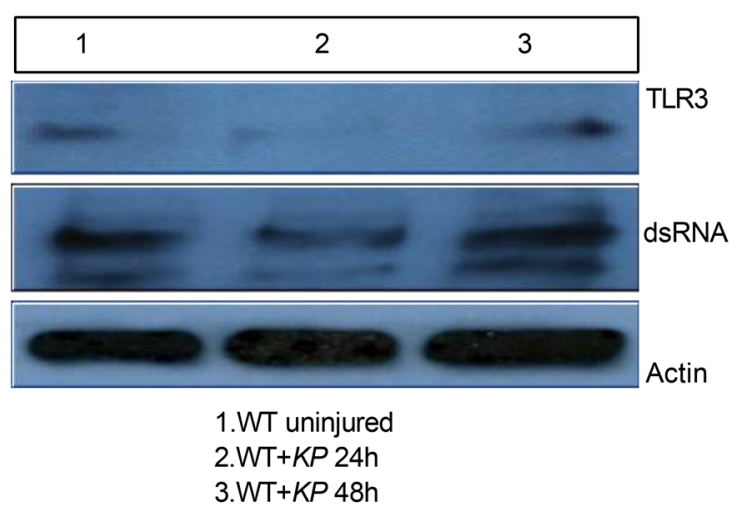

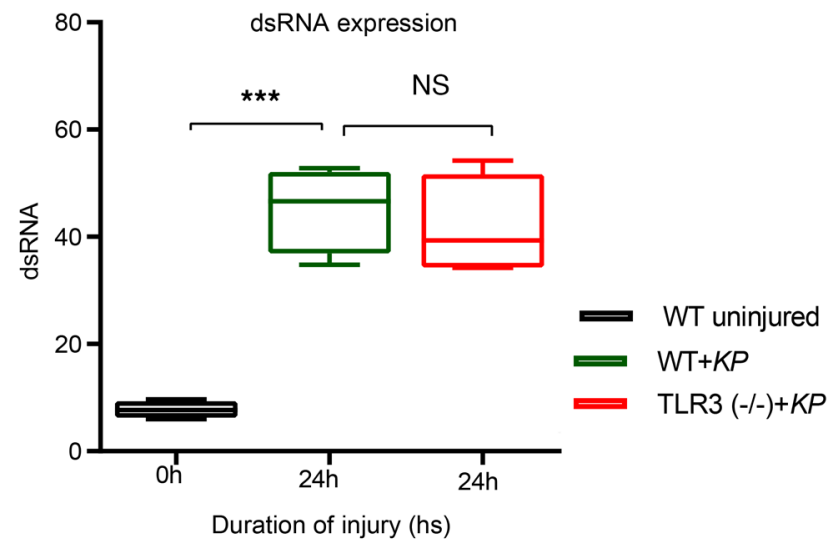

TLR3/dsRNA inhibitor
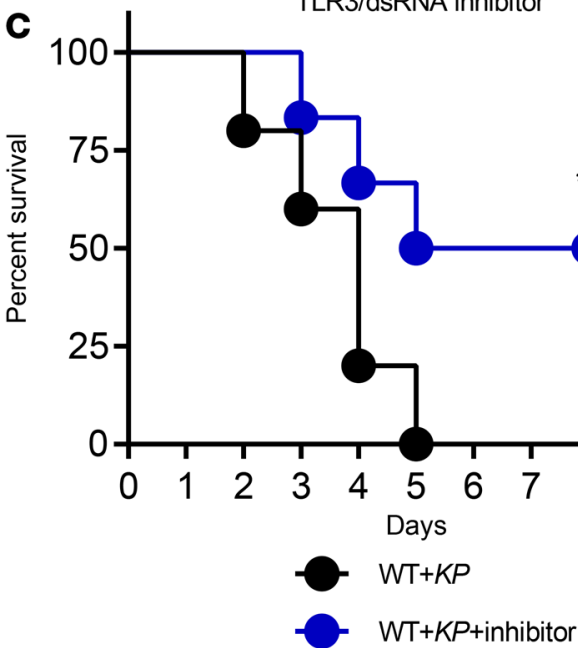

Figure 6. Increased survival with inhibition of TLR3 following pneumonia. WT and TLR3 $3^{-1-}$ were subjected to pneumonia, and the TLR3 and dsRNA expression were measured. Intracellular staining of TLR3 and flow cytometric analysis revealed significant upregulation of TLR3 in alveolar macrophages following KP administration. (A) A representative panel of flow cytometry data shown alongside pooled expression data. The lung lysate collected from uninjured and injured WT mice following KP (co-immunoprecipitation) (B) shows increased dsRNA expression ( $n=3$ per group). WT mice were administered TLR3/dsRNA complex inhibitor 1 hour before bacterial infection (C), which resulted in significantly reduced mortality following bacterial pneumonia ( $n=6$ per group). Statistical analysis was performed at each time point. Samples were analyzed using 1-way ANOVA with Tukey's multiple-comparisons test $\left({ }^{*} P<0.05 ;{ }^{* *} P<0.01 ;{ }^{* * *} P<0.001\right.$, WT vs. $T L R 3^{-/-}$mice). The box plots depict the minimum and maximum values (whiskers), the upper and lower quartiles, and the median. The length of the box represents the interquartile range.

It was important to establish how TLR3, as a viral sensor, is involved in the inflammatory response to gram-negative bacteria. Alveolar macrophages showed increased TLR3 expression following $K P$ compared with uninjured mice. We have previously published data that show dsRNA is significantly increased in serum and BAL of animals following LC and that a TLR3 dsRNA ligand inhibitor resulted in a reduction in lung injury and inflammation (4). The mortality in WT mice was partially reversed by the administration of TLR3/ dsRNA complex inhibitor following pneumonia. These experiments highlight the important role of dsRNA in TLR3-driven lung injuries, including LC and bacterial pneumonia. We report that TLR3 is an endogenous sensor of necrosis and that in its absence, there is a reduction in the amplification of the inflammatory response following LC (4). The current work provides further evidence that the dsRNA, likely released from necrotic cells, is the ligand for activation of TLR3 in KP. This finding has significant therapeutic implications. Other TLRs (e.g., TLR2/4) have been reported to play a key role in the generation of the acute inflammatory response following LC $(37,38)$. However, significant complications from uncontrolled gram-positive and -negative bacterial infections have been observed when the activities of these TLRs have been blocked (39-42).

It has been previously reported that the presence of alveolar macrophages in the resolution or progression of KP infection (6) and in animal models of Streptococcus pneumonia (43) is crucial in regard to the acute 
A Phagocytosis

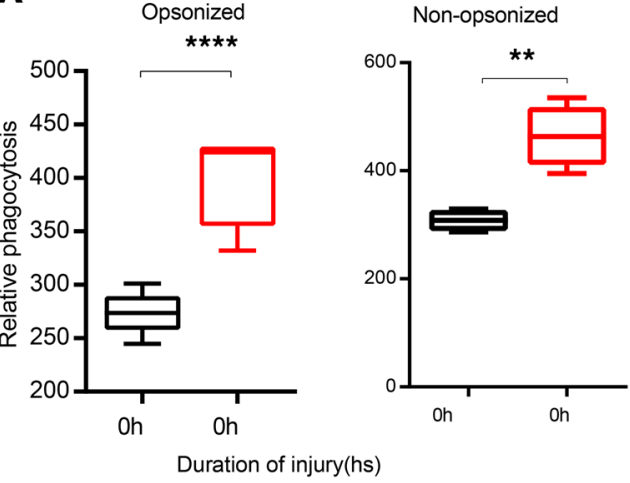

$\longrightarrow \mathrm{WT}+\mathrm{KP}$

TLR3(-I-) $+K P$

C
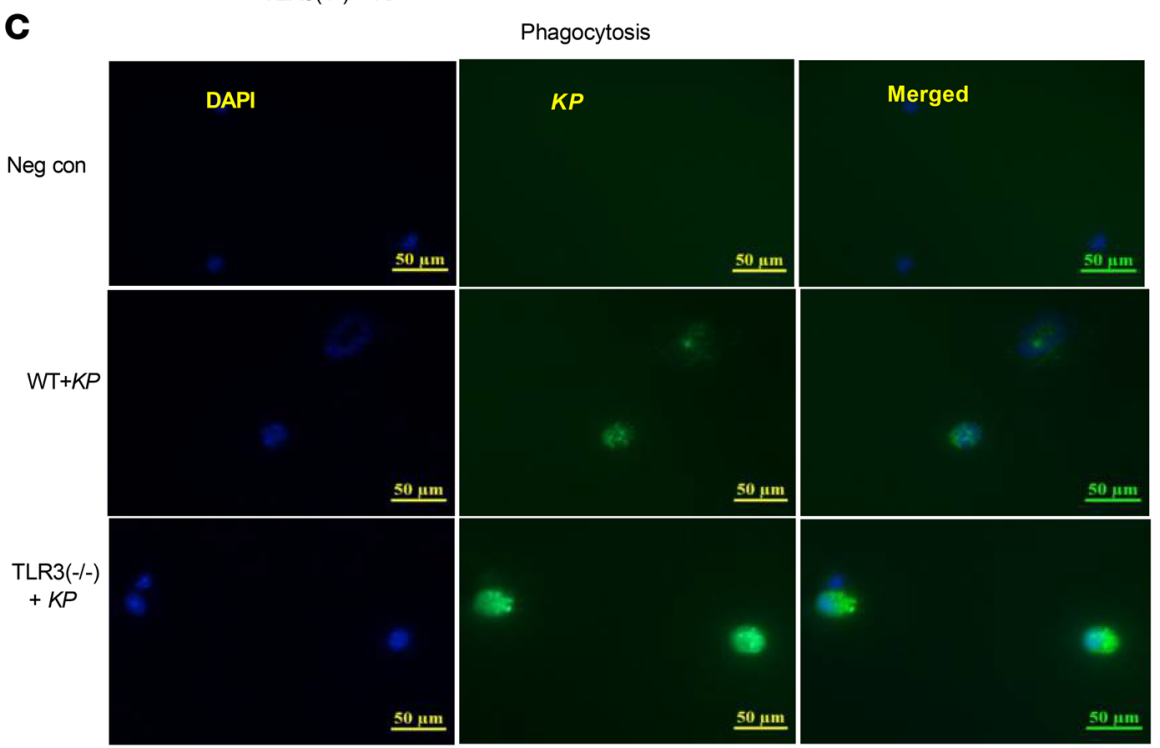

D

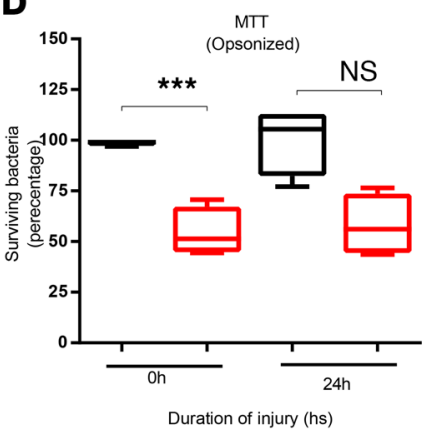

B
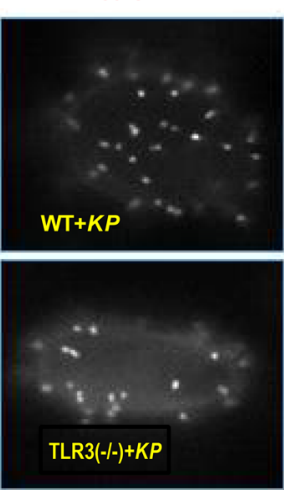

Phagocytosis

$$
\text { Bright field }
$$
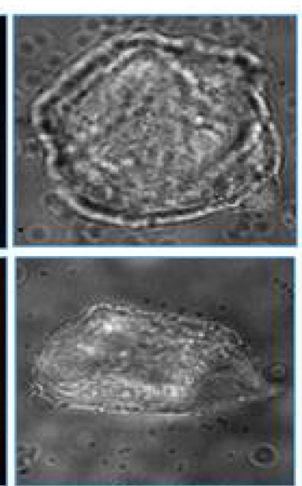

Overlay

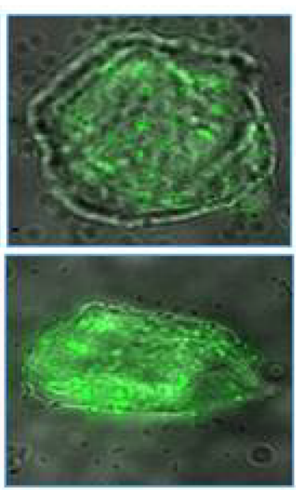

E
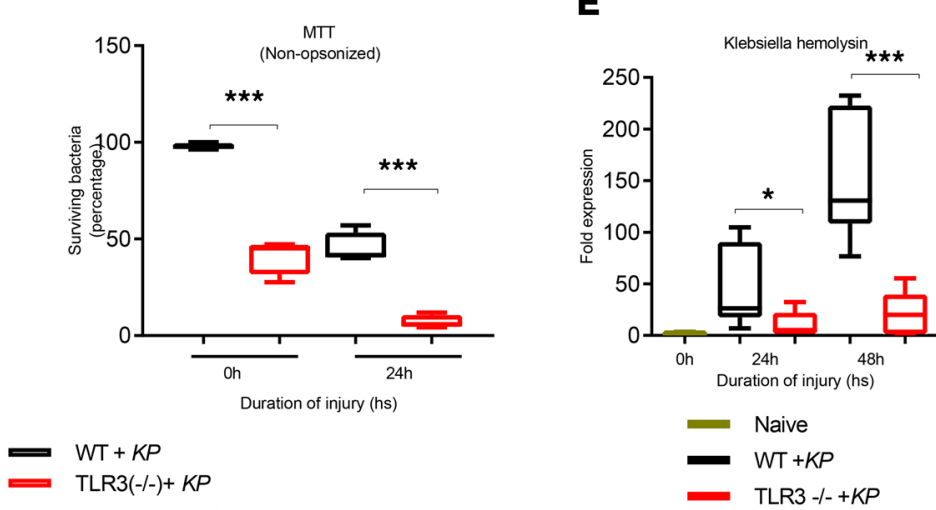

Figure 7. TLR3-/- mice alveolar macrophages show enriched phagocytic and bactericidal activity: phagocytosis. (A) Alveolar macrophages from WT and $T L R 3^{-/-}$uninjured and injured (KP-infected) mice were treated with FITC-labeled KP. Using a phagocytic assay (serum-opsonized and nonopsonized), relative phagocytic activity was measured ( $n=6$ per group). (B) Single-cell imaging of alveolar macrophages isolated in the presence and absence of $K P$ infection that were treated with FITC-labeled dead $K P$ with WT and TLR $3^{-1-}$ mice ( $n=3$ per group). (C) Macrophages were harvested from WT and $T L R 3^{-/-}$mice at 24 hours following live $K P$ administration and subjected to immunofluorescence staining with anti-Klebsiella pneumonia antibody ( $n=$ 3 per group). (D) MTT bacterial viability assay: The bactericidal activity in isolated alveolar macrophages from both naive and $K P$ administered mice was assessed (with serum and non-serum opsonized) ( $n=4$ per group). (E) The Klebsiella hemolysin gene (Khe) expression in real-time PCR: Lung RNA was isolated from WT and $T L R 3^{-/-}$mice following pneumonia, and Khe gene expression was measured ( $n=6$ per group from 2 separate experiments) Statistical analysis was performed at each time point. Samples were analyzed using 2-tailed unpaired $t$ test with Welch's $\operatorname{correction}\left({ }^{*} P<0.05^{* *} P<0.01\right.$; ${ }^{* * *} P$

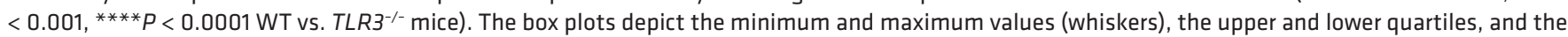
median. The length of the box represents the interquartile range. 
A

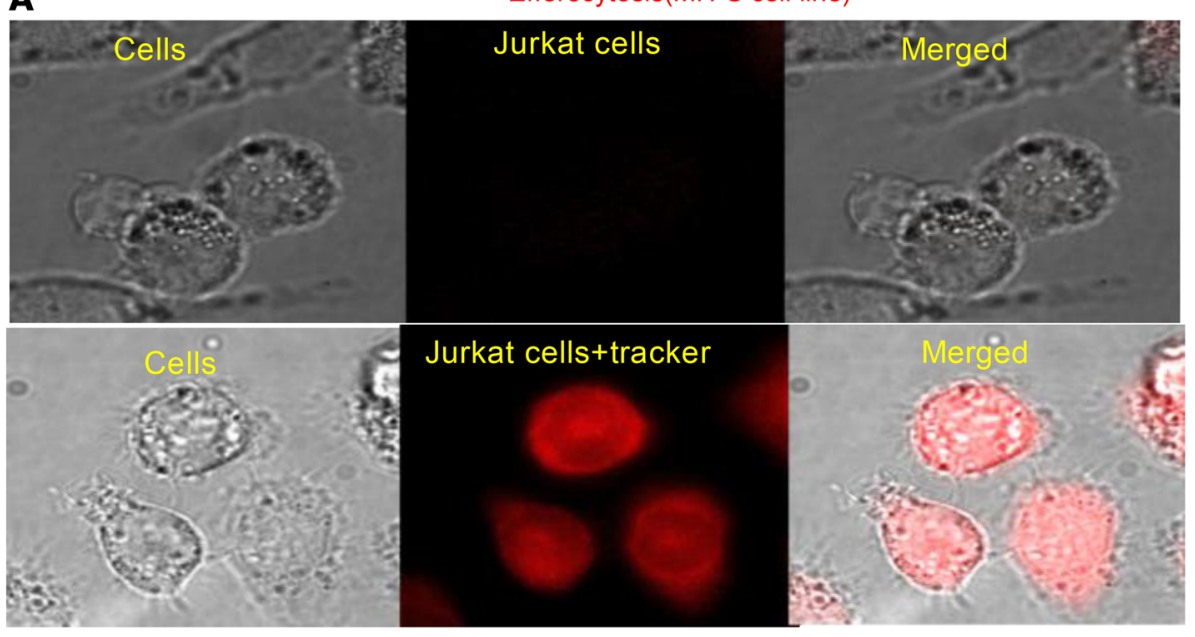

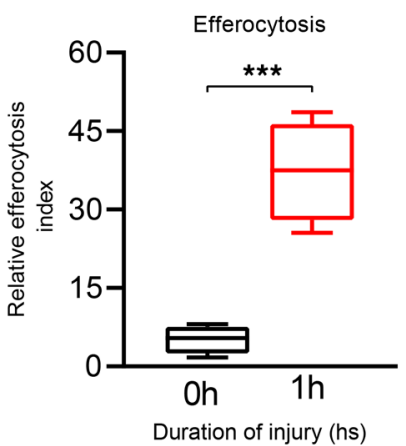

Cells only

Cells+cell tracker

B

WT mice

Macrophages

TLR3(-/-) mice
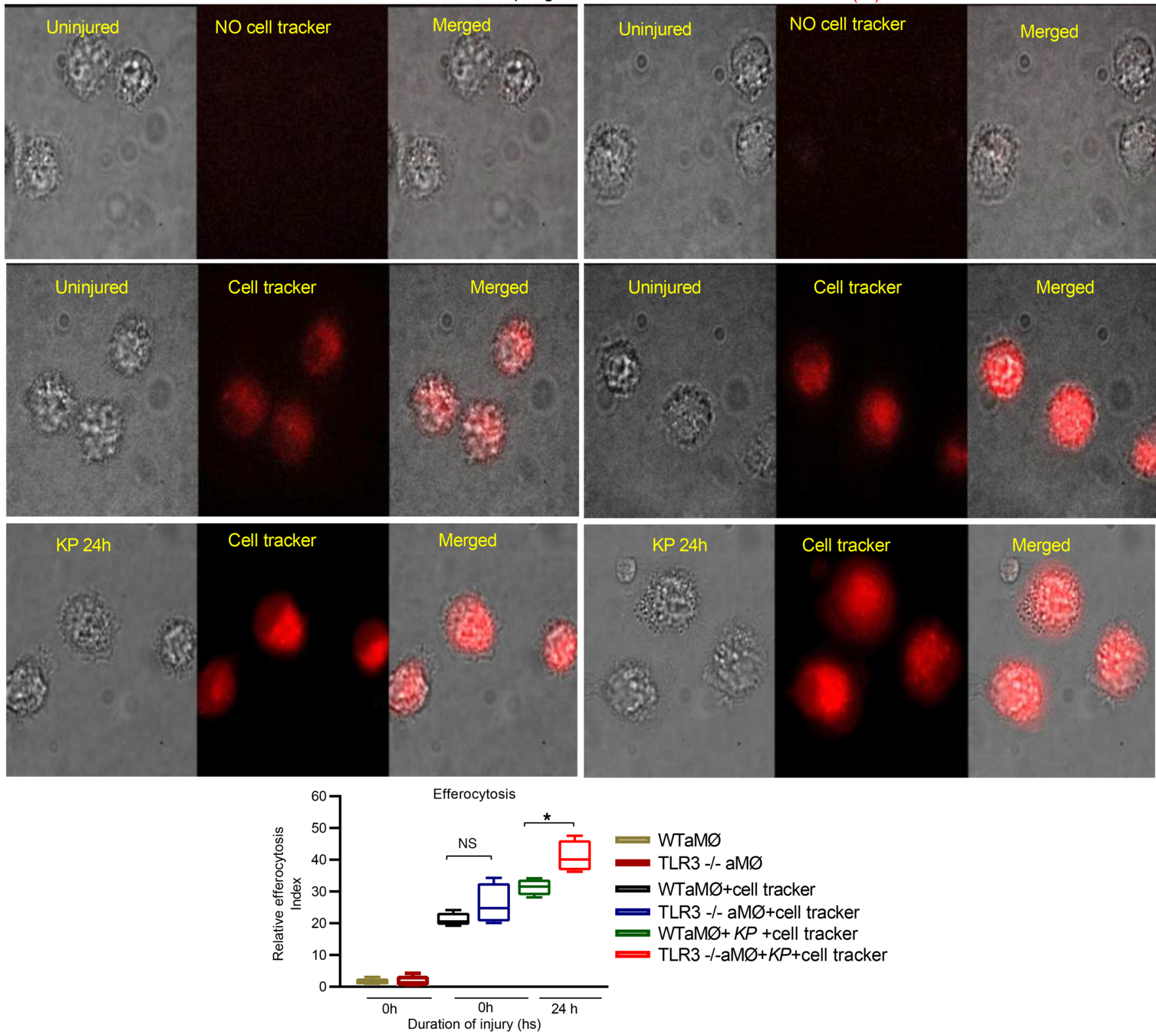

WTaMØ

TLR3 - - aM $\varnothing$

WTaM $\varnothing+$ cell tracker

— TLR3 -/- aM $\varnothing+$ cell tracker

WTaM $\varnothing+K P+$ cell tracker

TLR3 -/-aM $\varnothing+K P+$ cell tracker 
Figure 8. Improved macrophage efferocytosis in the absence of TLR3 following KP. (A) Representative figure of mouse macrophages cell line incubated, in the presence and absence of Cell Tracker orange, with apoptotic Jurkat (camptothecin-treated human Jurkat T cell line) cells and measures of the macrophage efferocytosis ( $n=4$ per group) ( ${ }^{* *} P<0.001$ untreated cells vs. treated cells). (B) Representative picture of alveolar macrophages from uninjured and injured (KP-infected) WT and $T L R 3^{-/-}$mice treated with Cell Tracker orange-labeled apoptotic Jurkat cells and with the measured fluorescence intensity. Fluorescence recovery analysis was done using ImageJ ( $n=4$ per group). Statistical analysis was performed at each time point. Samples were analyzed using 2-tailed unpaired $t$ test with Welch's correction ( ${ }^{*} P<0.05$ WT vs. TLR $3^{-1-}$ mice). The box plots depict the minimum and maximum values (whiskers), the upper and lower quartiles, and the median. The length of the box represents the interquartile range.

inflammatory response. Additionally, it has been shown that the polysaccharides of the capsule of $K P$ can affect phagocytosis by alveolar macrophages $(21,44)$. We hypothesized that deletion of TLR3 could aid in the resolution of $K P$ by possibly abrogating this effect. We evaluated the bactericidal and phagocytic capacity in isolated alveolar macrophages from both naive and injured $(K P)$ mice. The alveolar macrophages from $T L R 3^{-1-}$ mice showed enhanced phagocytic capacity and increased bactericidal activity as confirmed by an MTT bacterial viability assay (see methodology in refs. 45, 46 and in Figure 7D). Additional evaluation of Khe indicative of surviving $K P$ within the alveolar macrophages supported these findings. These results confirm the hypothesis that although TLR3 is a crucial PRR for viral infections, its deletion can lead to enhanced clearance of $K P$. Finally, the adoptive transfer experiments show that TLR3 deletion modulates the alveolar macrophage population, resulting in enhanced bacterial clearance of $K P$.

Though the bulk of this study focused on the alveolar macrophages, neutrophils, in addition to alveolar macrophages, are important effectors of bacterial clearance resulting from oxidant generation and formation of extracellular traps involved with bacterial killing (47). We observed an increase in neutrophil counts in the lung circulation at the 48-hour time point following $K P$ infection in $T L R 3^{-1-}$ mice. However, the neutrophils obtained from $T L R 3^{--}$mice showed a moderate reduction in oxidant production (NO) and oxidative stress (malondialdehyde) (Figure 11). A review of the literature suggests that peripheral neutrophils do not express TLR3 $(26,27)$. Therefore, it is possible that neutrophils are solely stimulated indirectly by increased chemokine generation during alveolar macrophage activation in the absence of TLR3. We observed that the splenic tissue from the $T L R 3^{--}$and WT mice had no difference in neutrophil infiltration by histology or MPO levels. Therefore, it is likely that the spleens did not contribute to the increased neutrophils observed with $T L R 3^{-1-}$.

This study has some limitations. The number of postmortem samples for the study was limited, and with no previous data on this subject, it would have been difficult to study the phenomenon of upregulation of TLR3 in patients with bacterial pneumonia prospectively. Additionally, the cellular mechanisms involved in the macrophage activity with the deletion of TLR3 need further elucidation.

In summary, our data indicate that TLR3 deletion is integral to improved bacterial clearance of $K P$. This action is directly mediated through increased phagocytic and bactericidal activity of the alveolar macrophages. Our findings are directly relevant to understanding and treatment of human disease. Neutralization of TLR3 has the potential not only to reduce lung injury associated with LC (4) but also to significantly reduce the severity of secondary bacterial pneumonia without off-target side effects. In contrast, significant complications from uncontrolled bacterial infections have been observed with specific neutralization of TLR2 and -4 (42), which are PRRs known to play a key role in the pathogenesis of bacterial infections.

\section{Methods}

Human lung histology and immunofluorescence staining. Human LC specimens were obtained from the All India Institute of Medical Sciences (India), and access to these tissue specimens was approved by the local research ethics committee. For the lung specimens, slides of lung tissue from autopsy samples were obtained from 10 patients who died following $K P$. The formalin-fixed control and lung-contused sections were paraffin-embedded, sectioned, and stained with H\&E. The sections that represented histological changes consistent with progression of lung contusion in human subjects were further analyzed for IHC localization of TLR3. Briefly, 5- $\mu \mathrm{m}$ sections were deparaffinized and rehydrated. Sections were washed and blocked for 15 minutes, incubated overnight at $4^{\circ} \mathrm{C}$ with polyclonal TLR3 antibody (1:100, PA5-29619, eBioscience), and incubated with goat anti-rabbit Alexa Fluor 594 antibody (1:2000, A-11037, BioLegend) for 60 minutes at room temperature (RT). Sections were mounted using Pro Long Gold containing DAPI (Invitrogen, Thermo Fisher Scientific). Cells on coverslips were then mounted in mounting medium, and photomicrographs of the invasive sections were analyzed digitally using Photoshop software version 9.0.2 (Adobe) (4). 
A

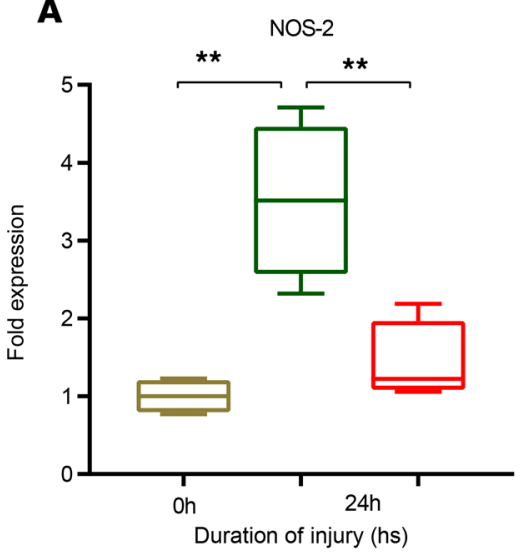

D

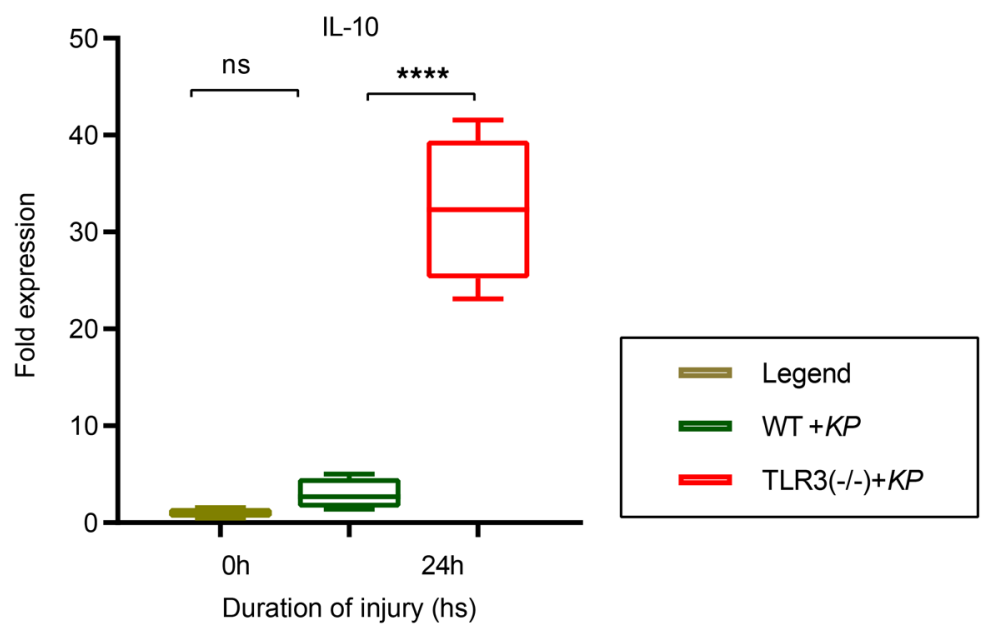

TLR3-I- compared to WT mice RNA seq. from isolated aMФ chemokine signaling

\section{B}

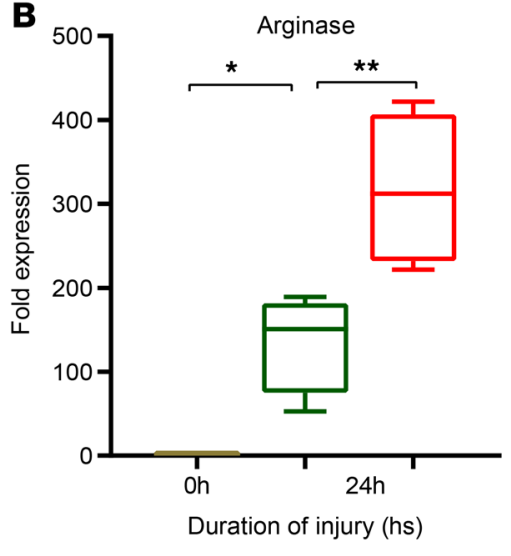

Duration of injury (hs)

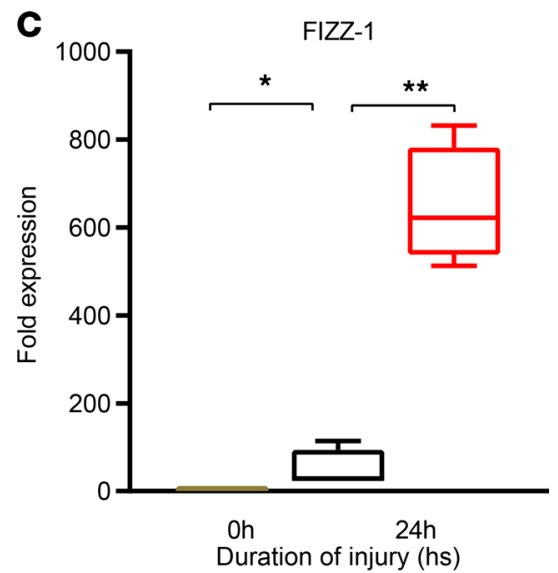

Figure 9. The deletion of TLR3 increased chemokine production from alveolar macrophages following KP. Characterization of BAL macrophages from WT and $T L R 3^{-/-}$mice following pneumonia and the levels of NOS-2 (A), arginase-1 (B), FIZZ-1 (C), and IL-10 (D) genes were measured by quantitative reverse transcriptase PCR $(n=5$ per group). WT and $T L R 3^{-/-}$mice were subjected to $K P$, and RNA was isolated from the BAL macrophages. The box plots depict the minimum and maximum values (whiskers), the upper and lower quartiles, and the median. The length of the box represents the interquartile range. (E) RNA-sequencing analysis of alveolar macrophages reveals that the $\mathrm{TLR}^{-/-}$mice produced significantly higher amounts of chemokines, particularly CXCL1 (KC) and CXCL2 (MIP-2) ( $n=6$ per group). Statistical analysis was performed at each time point. Samples were analyzed using 1-way ANOVA with Tukey's multiple-comparisons test $\left({ }^{*} P<0.05 ;{ }^{* *} P<0.01 ;{ }^{* *} P<0.001\right.$; ${ }^{* * * *} P<0.0001$, WT vs. TLR3 ${ }^{-/-}$mice). Image credit: Advaita Corporation @ 2017.

\section{3}

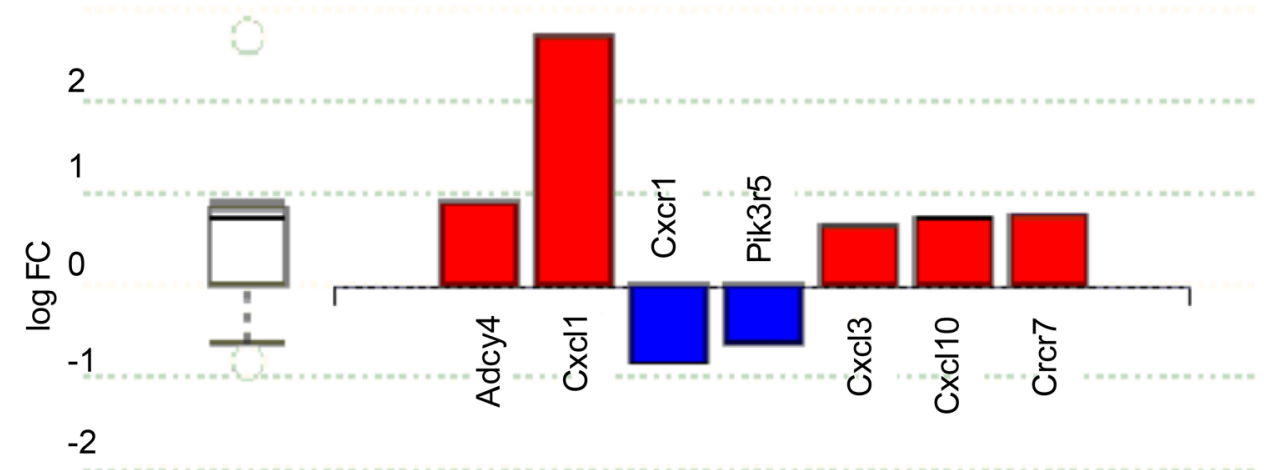

$-3$

(c) Advaita Corporation 2017 
A
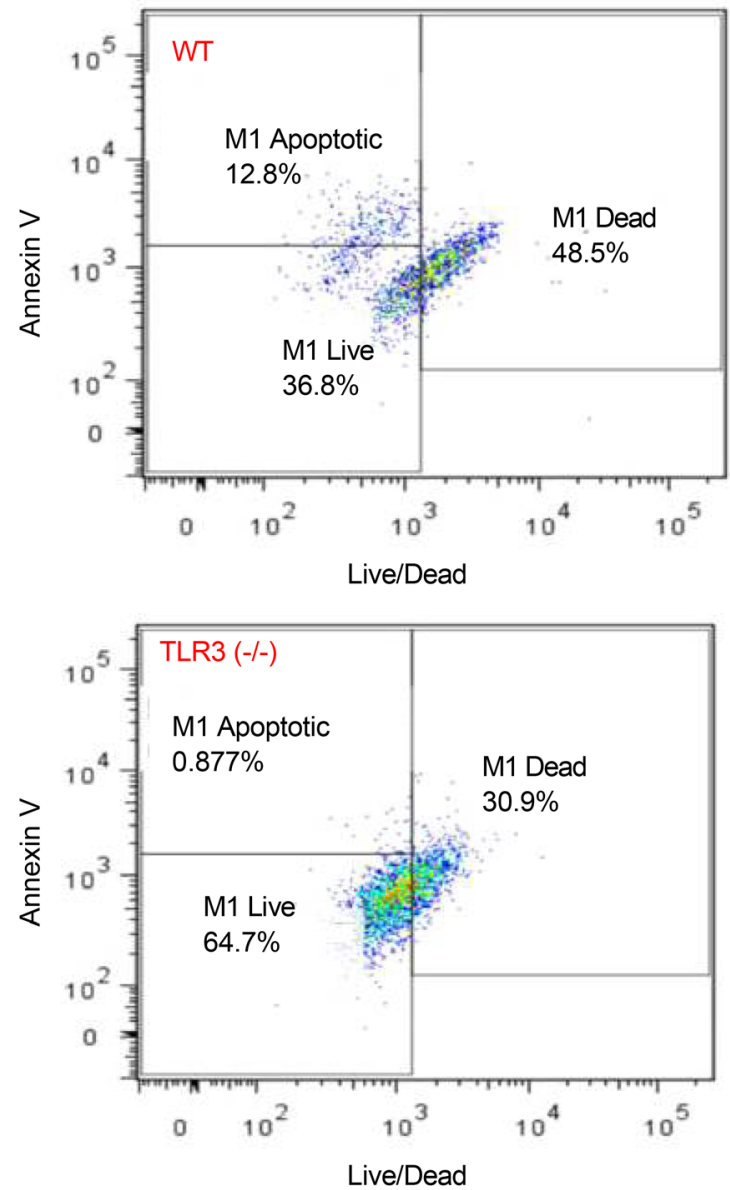

B

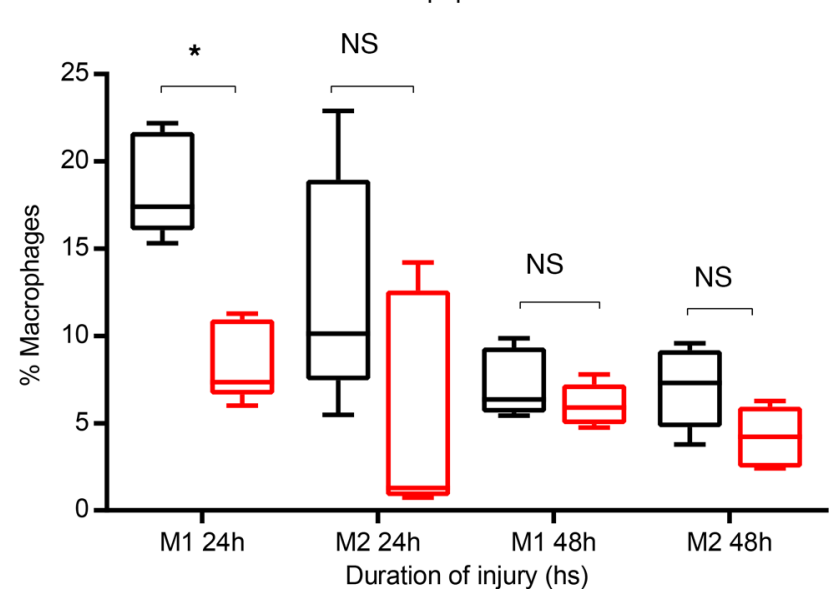

K.pneumoniae (24h)
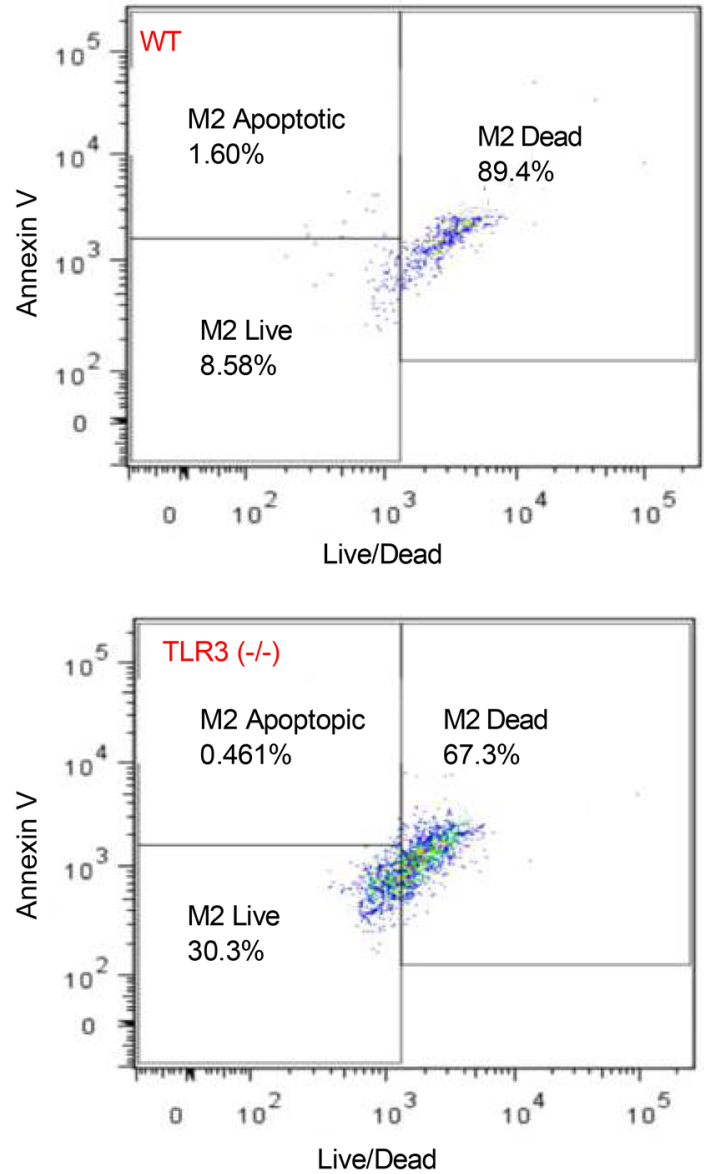

Live/Dead

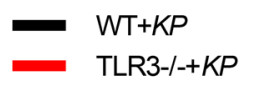

Figure 10. Increased alveolar macrophage apoptosis in WT compared with $T L R 3^{-/-}$mice following primary and secondary pneumonia. (A and B) Characterization of BAL cells using flow cytometry revealed that the degree of M1 and M2 macrophage apoptosis was higher in WT mice following KP infection compared with $T L R 3^{-/-}$mice ( $n=5$ per group). Statistical analysis was performed at each time point. Samples were analyzed using 2 -tailed unpaired $t$ test with Welch's correction ( ${ }^{*} P<0.05 \mathrm{WT}$ vs. TLR3 ${ }^{-/-}$mice). The box plots depict the minimum and maximum values (whiskers), the upper and lower quartiles, and the median. The length of the box represents the interquartile range.

Mouse TLR3 immunocytochemistry. Macrophages were added $\left(3 \times 10^{5}\right)$ to serum-coated, chambered, 8-well tissue culture plates and allowed to adhere at RT for 1 hour. Cells were washed with PBS. Cells were fixed and the fixative was removed by aspiration and incubated in blocking buffer. To detect TLR 3 expression, macrophages were permeabilized with $0.3 \%$ Triton X-100 for 10 minutes at RT before incubation 

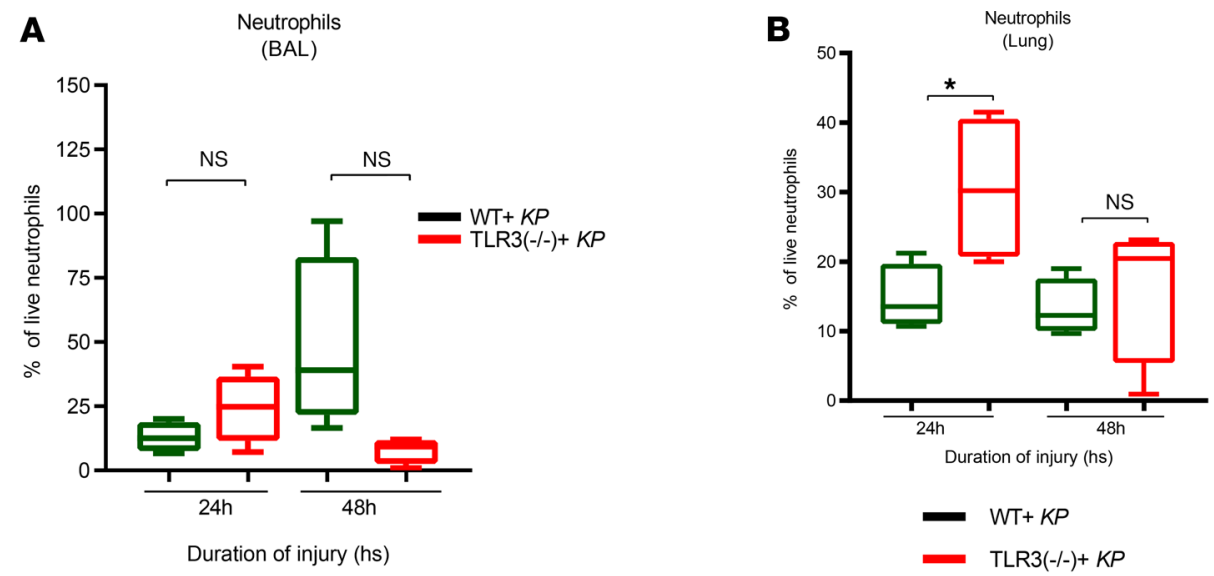

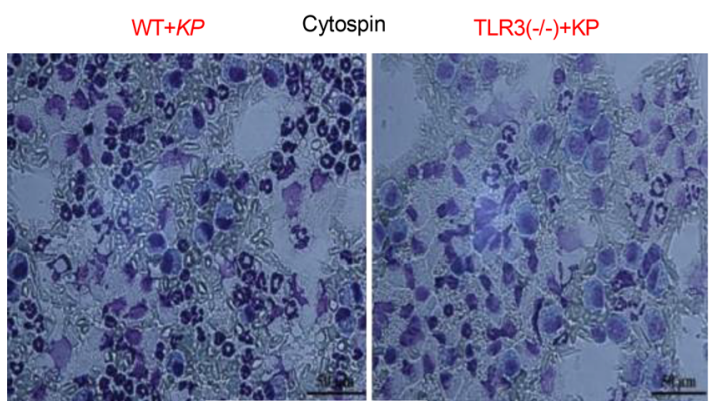

BAL- neutrophils-24h

C

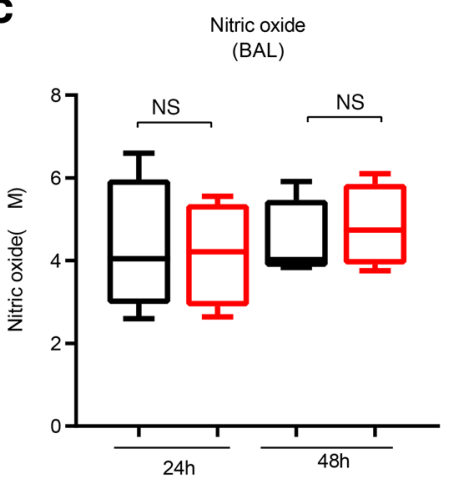

Duration of injury (hs)
D

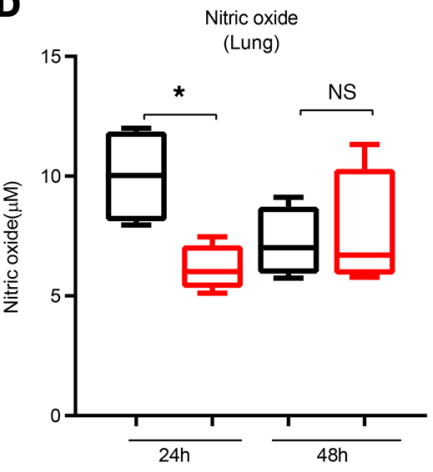

Duration of injury (hs)

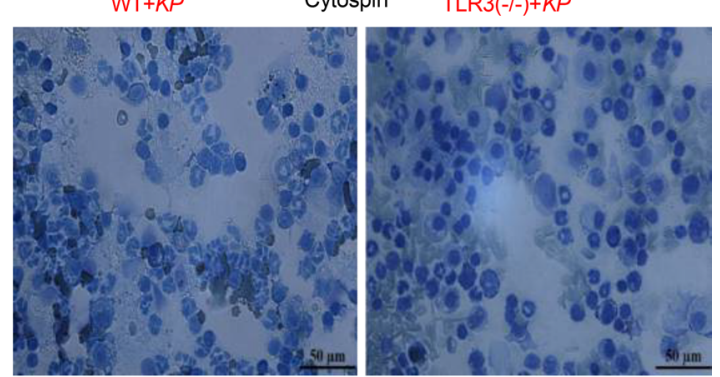

Lung-neutrophils-24h

$\mathbf{E}$

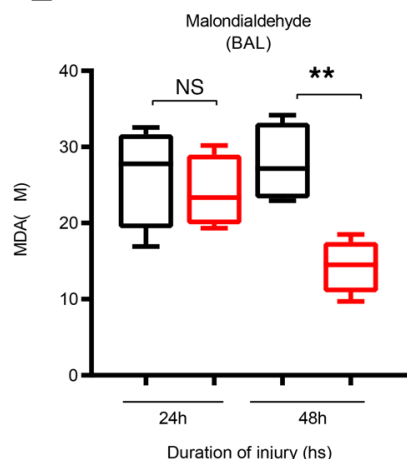

WT $+K P$
TLR3(-/-)+KP

Figure 11. Role of neutrophils following pneumonia. The WT and $T L R 3^{-/-}$mice treated with pneumonia and the BAL and lung neutrophil level measured by cytospin method. (A) No significant neutrophil difference in the BAL between WT and TL3 $3^{--}$mice. (B) The levels of neutrophils were significantly higher in the WT mice lungs compared with the $T L R 3^{-/-}$mice. (C and $\left.\mathbf{D}\right)$ The level of NO was measured in the BAL and lung of WT mice and TLR $3^{-/-}$mice following pneumonia. (E) The levels of MDA were significantly higher in WT mice at the 48-hour time point compared with $T L R 3^{-1-}$ mice following $K P$ ( $n=6$ per group, 2 independent experiments). Statistical analysis was performed at each time point. Samples were analyzed using 2-tailed unpaired $t$ test with Welch's correction ${ }^{*} P<0.05$ WT vs. $T L R 3^{-1-}$ mice). The box plots depict the minimum and maximum values (whiskers), the upper and lower quartiles, and the median. The length of the box represents the interquartile range.

with TLR3 polyclonal antibody (1:100, MAB3005, R\&D Systems) for 1 hour at RT and then incubated with goat anti-rabbit Alexa Fluor 594 (1:2000, A-11037, BioLegend) for 60 minutes at RT. Sections were analyzed digitally using Photoshop software version 9.0.2.

Animals. Male and female age-matched (6-8 weeks, bred in-house) C57BL/6 and TLR3 $3^{-1-}$ (The Jackson Laboratory) mice were used.

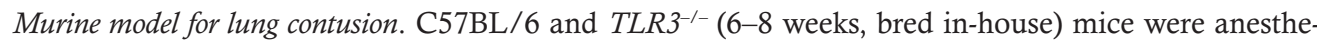
tized, and LC was induced. Briefly, after induction of anesthesia, each mouse was placed in a left lateral 


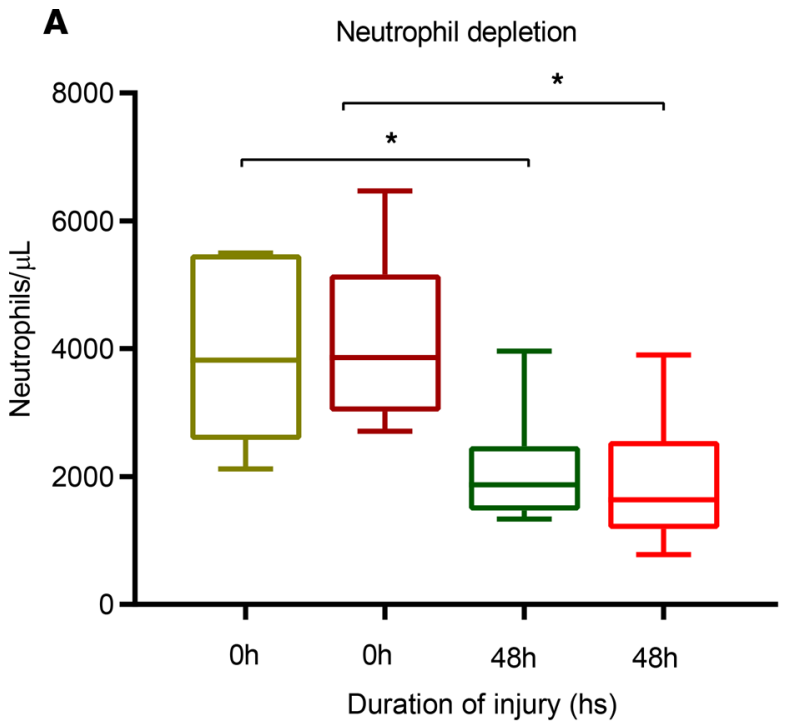

WT uninjured
TLR3-/- uninjured
WT+Ly6G Ab
TLR3 -/- +LY6G Ab
B

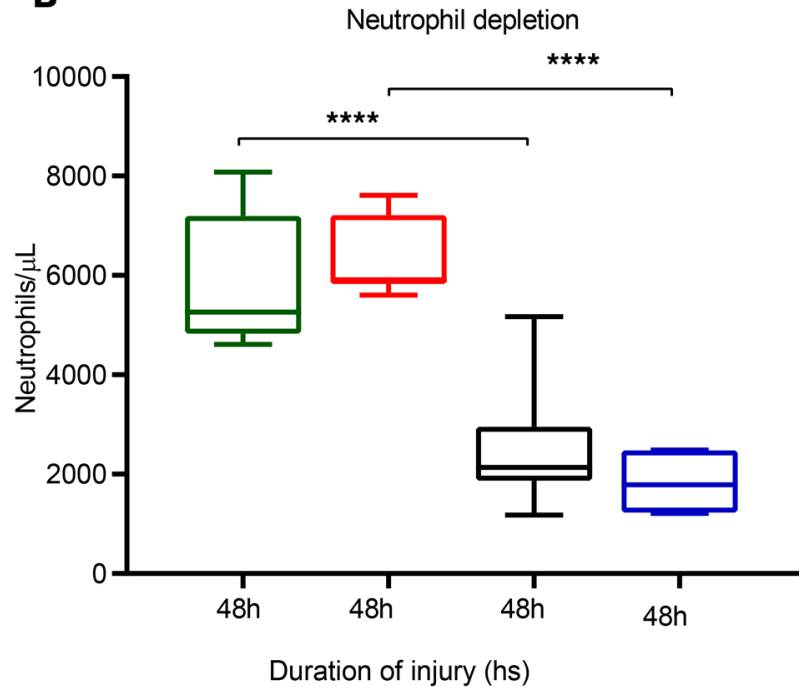

$\begin{array}{ll}= & \text { WT }+K P \\ & \text { TLR3-I-+KP } \\ & \text { WT+Ly6G Ab+KP } \\ = & \text { TLR3-/-+Ly6G Ab+KP }\end{array}$

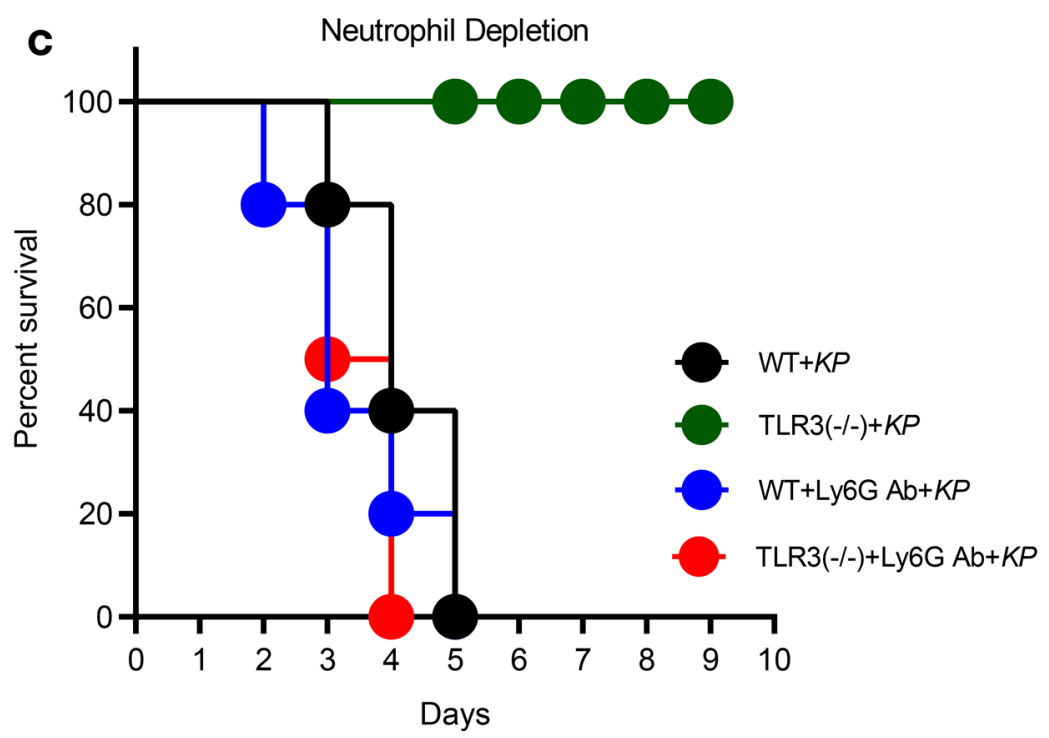

Figure 12. Neutrophils play a critical role in bacterial clearance, phagocytosis, and killing following KP. WT and TLR3 $3^{-/-}$mice received anti-Ly6G antibody (source and catalog/clone number) (i.p.) to deplete the number of neutrophils. (A) After 48 hours, blood was collected, and the hematological parameters were examined in the mice. (B) WT and $T L R 3^{-1-}$ mice were administered $K P$ (500 CFU), and 48 hours after $K P$, blood was collected. The hematological parameters were examined in the mice. (C) In our survival study, the antibody-administered WT and TLR3 ${ }^{-/}$mice both died in similar patterns as the WT mice $K P$ alone once the neutrophils were depleted ( $n=6$ per group, 2 independent experiments). Statistical analysis was performed at each time point. Samples were analyzed using 1 -way ANOVA with Tukey's multiple-comparisons test ( ${ }^{*} P<0.05{ }^{* * * *} P<0.0001$, WT vs. TLR $3^{-1-}$ mice). The box plots depict the minimum and maximum values (whiskers), the upper and lower quartiles, and the median. The length of the box represents the interquartile range.

position and injured with a cortical contusion impactor. The right chest was struck along the posterior axillary line $1.3 \mathrm{~cm}$ above the costal margin with a velocity of $5.8 \mathrm{~m} / \mathrm{s}$ adjusted to a depth of $10 \mathrm{~mm}(4,24)$.

Administration of anesthetic, analgesic, and resuscitation. Animals were anesthetized by intraperitoneal (i.p.) injection of ketamine ( $80-120 \mathrm{mg} / \mathrm{kg}$ body weight) and xylazine (5-10 $\mathrm{mg} / \mathrm{kg}$ body weight) or isoflurane inhalation. Systemic analgesics were not used because of their effects on the immune/ 


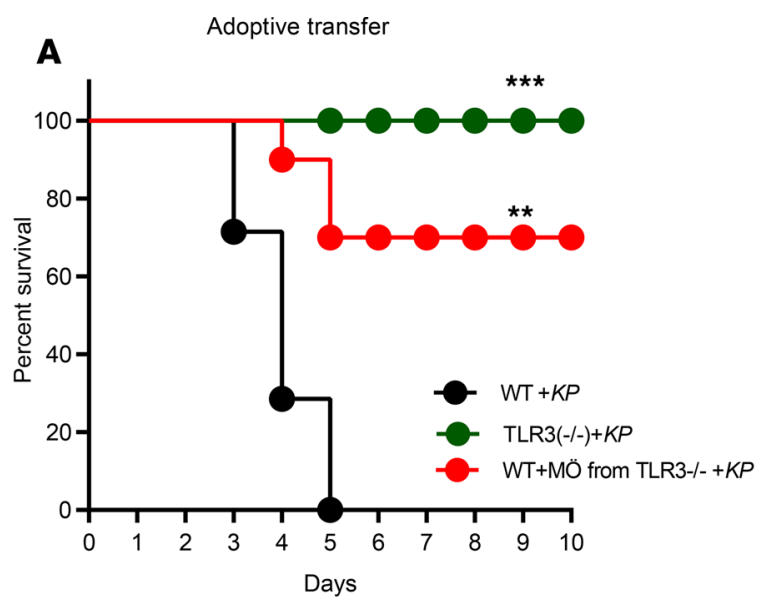

Figure 13. Adoptive transfer of $\mathrm{TLR3}^{-/-}$macrophages restores host defense in WT mice following $K P$ infection. (A) Macrophages from $T L R 3^{-/-}$mice were adoptive transferred into WT mice; 48 hours later, the mice were inoculated (via deep oral hypopharyngeal groups) with 500 CFU of $K P$, and mortality was monitored up to 10 days $\left({ }^{*} P<0.05\right.$ by log-rank test). Data shown represent $n=10$ mice per group from 2 independent experiments. (B and $\mathbf{C}$ ) Representative pictures of blood and lung culture following pneumonia. WT mice had reduced blood and lung CFU at 24 and 48 hours following $T L R 3^{-/-}$macrophage administered mice compared to $\mathrm{WT}+K P$ only mice ( $n=6$ mice per group, 2 independent experiments). Statistical analysis was performed at each time point. Samples were analyzed using 2-tailed unpaired $t$ test with Welch's correction $\left({ }^{*} P<0.05\right.$; ${ }^{* *} P<0.01 ;{ }^{* *} P<0.001$, WT vs. TLR3 ${ }^{-1-}$ mice). The box plots depict the minimum and maximum values (whiskers), the upper and lower quartiles, and the median. The length of the box represents the interquartile range.

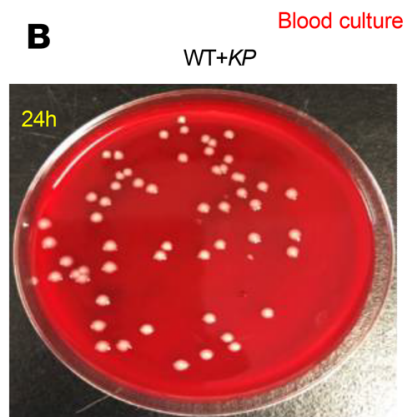

WT+MÖ from TLR3(-/-)+KP
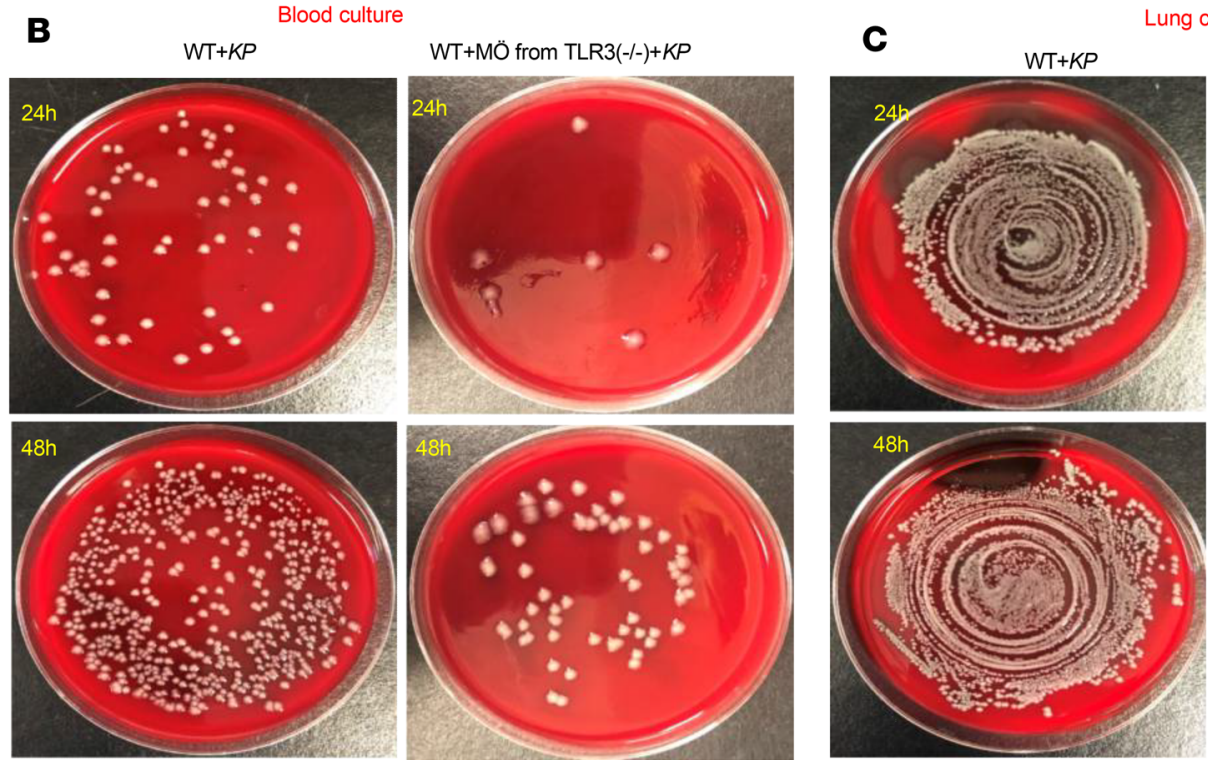

ung culture
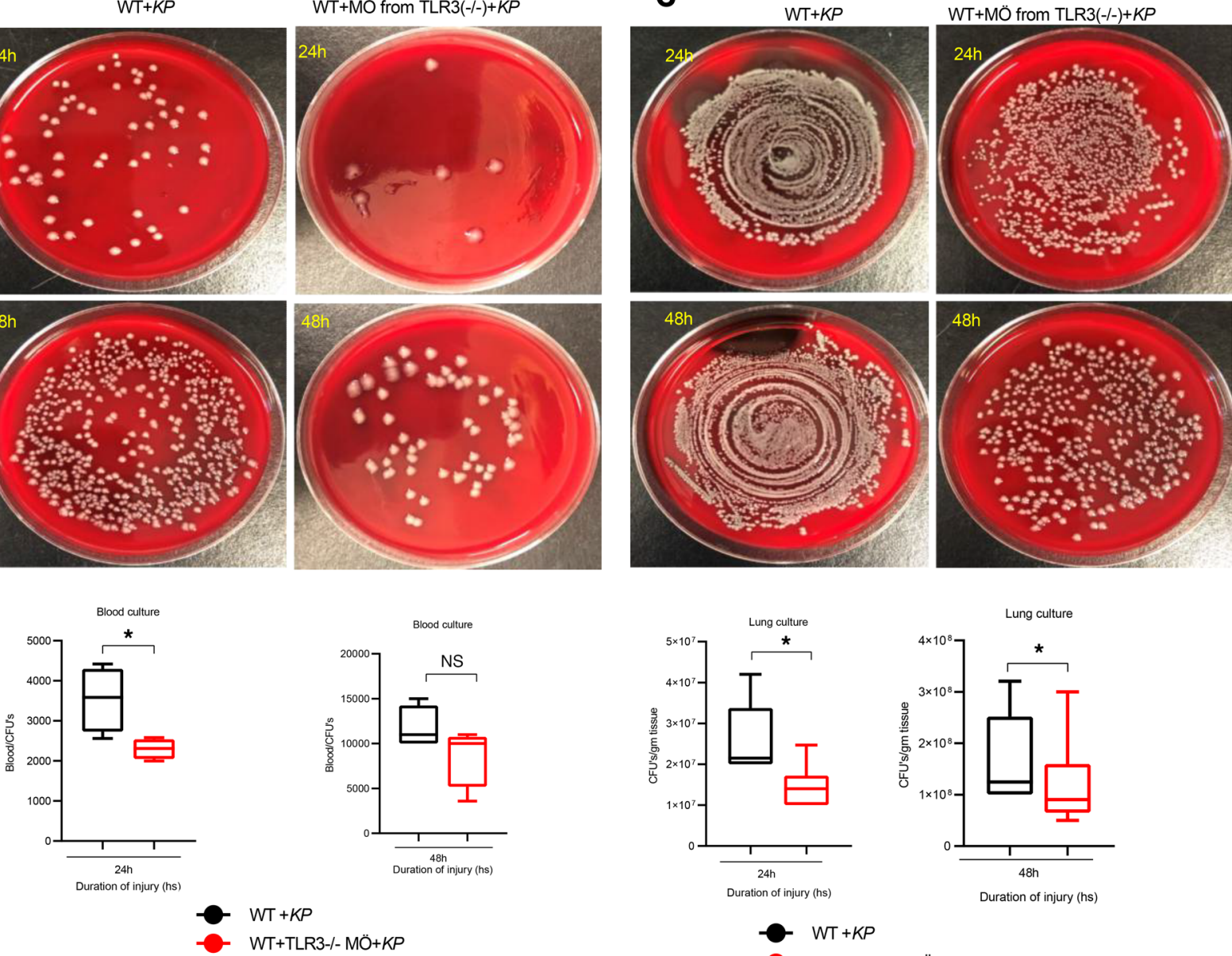

$W T+K P$
$W T+T L R 3-I-M O ̈+K P$
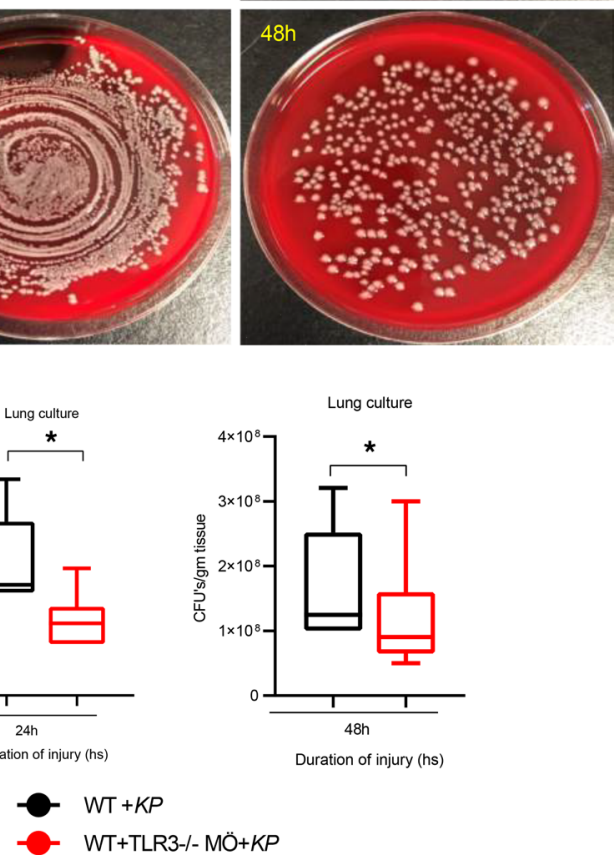

inflammatory system. In the event of severe respiratory distress beyond 48 hours, animals were humanely euthanized (48).

Bacterial pneumonia model. K. pneumoniae, strain 43816, serotype 2, was purchased from American Type Culture Collection (ATCC). This microbial strain was cultured overnight in trypticase soy broth (Becton Dickinson) with re-inoculation the following morning into fresh medium to bring the bacteria into a logarithmic growth phase. Optical density (OD) was measured at a wavelength of $600 \mathrm{~nm}$, and serial dilutions were then made to reach a concentration of $500 \mathrm{CFU}$ of bacteria per $30 \mu \mathrm{L}$. Mice were inoculated with the bacterial suspension or saline control via deep oral hypopharyngeal injection under isoflurane anesthesia. Animals were allowed to recover spontaneously, and survival data were recorded every 8 hours for 10 days $(5,49,50)$. 

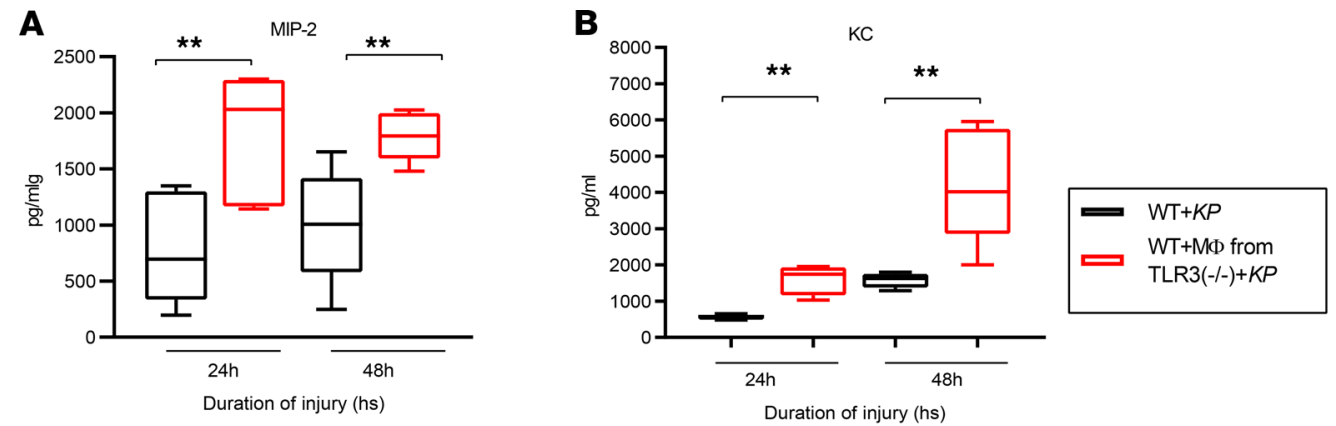

Histology-Adoptive transfer

\section{C}

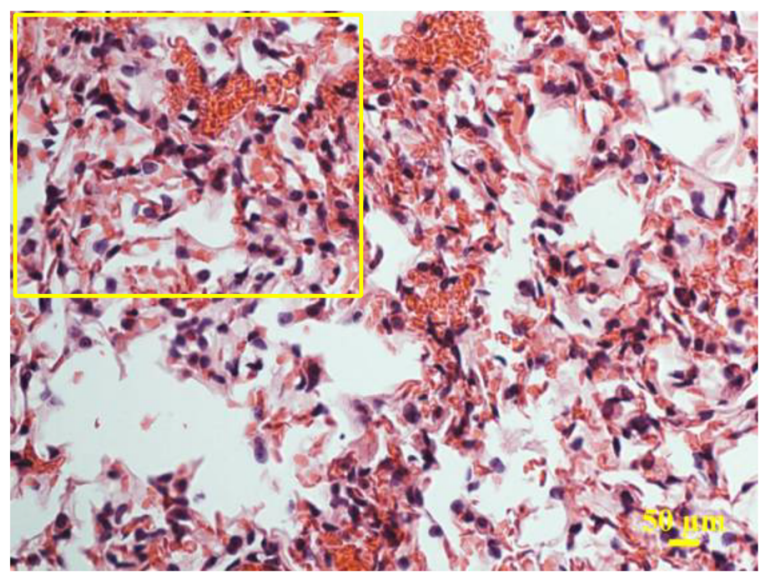

D $W T+M \Phi$ from TLR3(-I- $)+K P 24 h$
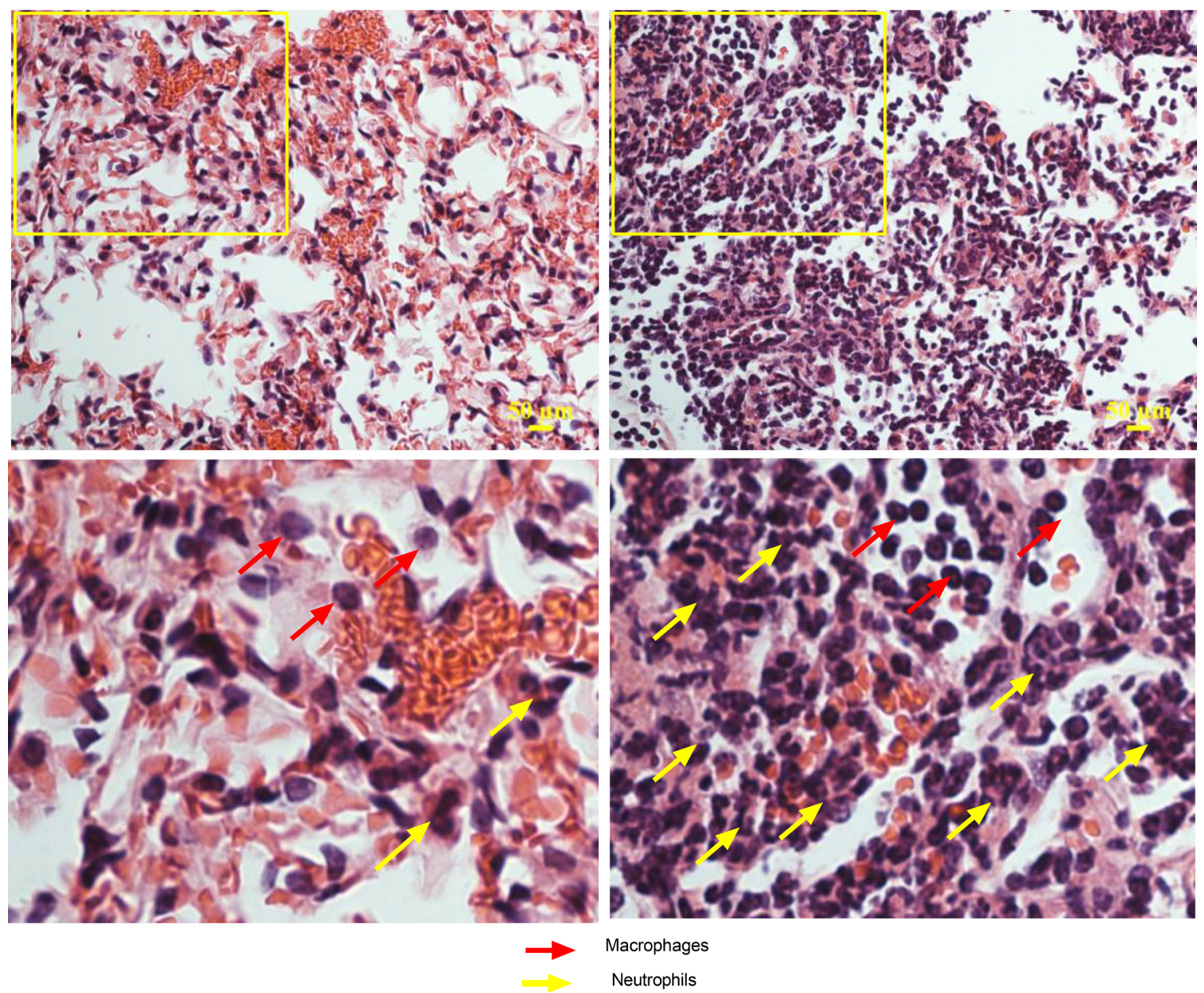

Figure 14. Adoptive transfer of $T_{L R 3^{-/-}}$macrophages shows increased neutrophils in WT mice following $K P$ infection. (A and B) Macrophages from $T L R 3^{-/-}$mice were adoptive transferred into WT mice; 48 hours later, the mice were inoculated with KP, and the expression of KC and MIP-2 were measured by ELISA ( $n=6$ mice per group). The levels of both cytokines were significantly elevated in the adoptive transferred WT mice. (C and D) Macrophages from $T L R 3^{-1-}$ mice were adoptively transferred into WT mice; 48 hours later, the mice were inoculated with $K P$. Lungs were collected at 24 hours after infection and stained with H\&E. Representative histological pictures are shown following pneumonia ( 3 mice per group) (original magnification, $\times 600)$. Statistical analysis was performed at each time point. Samples were analyzed using 2-tailed unpaired $t$ test with Welch's

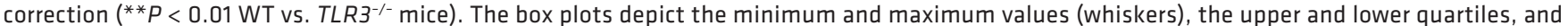
the median. The length of the box represents the interquartile range. 
Determination of bacterial load in lung, spleen, and blood. Bacterial loads in the lungs, spleen, and blood of WT and $T L R 3^{--1}$ mice administered with $K P$ were determined at 24- and 48-hour time points. Quickly, the lungs and spleen were removed and maintained in sterile conditions. A small aliquot $(100 \mu \mathrm{L})$ of tissue homogenate was serially diluted in sterilized PBS, plated on 5\% sheep blood agar, and incubated overnight at $37^{\circ} \mathrm{C}$, after which the number of colonies was determined. For blood CFU determinations, blood was collected via cardiac puncture using a sterile 18-gauge needle. To measure bacterial number, $100 \mu \mathrm{L}$ of undiluted blood was plated onto 5\% sheep blood agar plates (Thermo Fisher Scientific) and incubated at $37^{\circ} \mathrm{C}$, and then colonies were counted $(4,5,49)$.

Immunoneutralization of TLR3 in WT mice. TLR3 was blocked by a single injection of murine monoclonal antibodies against this receptor (50 and $100 \mu \mathrm{g}$ per mouse, MAB3005, R\&D Systems). The antibody injections were performed intraperitoneally, 48 hours before pneumonia. Mice survival was noted 10 days following pneumonia inoculation. Using a combination of LysoTracker and permeability assay, in previous studies we were able to show that the antibodies do enter the cell. In addition, we observed externalization of TLR3 to the surface of the cell (4). These 2 factors account for the efficacy of the antibody in the neutralization of TLR3.

Albumin concentrations in BAL. Albumin concentrations in the BAL were measured by ELISA using polyclonal rabbit anti-mouse albumin antibody (1:1000, A90-135, Bethyl Laboratories, Inc.) and HRP-labeled goat anti-rabbit IgG (1:5000, A90-134P, Bethyl Laboratories, Inc.) $(48,51)$.

Determination of cytokine levels in BAL and lungs. Soluble concentrations of IL-1 $\beta$, IL-6, MIP-2, KC, and MPO in the BAL and lung, following LC and pneumonia, were determined using ELISA. Antibody pairs (capture and biotinylated reporter antibody) and recombinant cytokines for these assays were obtained from R\&D Systems. These cytokines reflect the various attributes of the inflammatory response following pneumonia $(50,52)$.

Cytospin cell count. For cytospin preparations, BAL cells were centrifuged at $600 \mathrm{~g}$ for 5 minutes using a Cytospin II (Shandon Scientific), stained with Diff-Quik (Dade Behring Inc.) and analyzed by examination under a light microscope at original magnification $20 \times$ as described previously $(4,52)$.

Tetrazolium dye reduction assay of bacterial killing. The killing ability of alveolar macrophages after administration of pneumonia from WT mice and $T L R 3^{-1-}$ mice was quantified using a tetrazolium dye reduction assay (53). Results were expressed as a percentage of survival of ingested bacteria normalized to the percentage of control, where the A595 experiment values were divided by the average of the A595 control values.

Phagocytosis assay. Phagocytosis assays were performed as previously described $(4,16)$. Briefly, following administration of pneumonia, alveolar macrophages were isolated from BAL extracted from WT and $T L R 3^{-1-}$ mice. Cells were aspirated and replaced with $50 \mu \mathrm{L}$ serum-free medium. Macrophages were then incubated with FITC-labeled, heat-killed KP. Phagocytosis of FITC-labeled bacteria was measured after quenching of noningested bacteria with trypan blue. Additionally, single-cell imaging of alveolar macrophages with FITC-labeled dead $K P$, with phagocytosis of WT and $T L R 3^{-1-}$ mice, was performed.

Macrophage phagocytosis of live K. pneumonia. Phagocytosis of $K P$ by macrophages was measured by coordinated phagocytosis assays, which were performed with adherent macrophages. $K P$ were opsonized in fresh $5 \%$ (vol/vol) FBS for 30 minutes at $37^{\circ} \mathrm{C}$, washed in PBS, and resuspended in $\mathrm{RPMI} / \mathrm{H}$ at $5000 \mathrm{CFU} / \mathrm{mL}$. They were then added to 8 -well chambered plates and allowed to adhere at RT for 15 minutes. Samples were incubated at $37^{\circ} \mathrm{C}$ for the indicated times and then washed with cold PBS. Cells were fixed with 4\% paraformaldehyde for 10 minutes on ice, followed by 10 minutes at RT. Fixative was removed by aspiration, and samples were washed 3 times in PBS and incubated in blocking buffer ( $5 \%$ goat serum in PBS) for 30 minutes at RT. To detect ingested bacteria, cells were incubated with antibody specific for $K P$ (1:200, PA1-7226, Thermo Fisher Scientific) for 1 hour at RT and washed 3 times with washing buffer. Nuclei were stained with DAPI. Cells were then mounted on coverslips in mounting medium (Dako, Agilent), and photomicrographs of the invasive sections were analyzed digitally using Photoshop software version 9.0.2.

Ex vivo efferocytosis assay (flow cytometry method). Ex vivo efferocytosis assay was performed as previously described (54). For this experiment MH-S, a murine alveolar macrophage cell line, was received from ATCC. Alveolar macrophages were isolated from the WT and $T L R 3^{-/-}$mice following $K P$ administration. Macrophages were incubated with apoptotic targets (1:5) for 1 hour at $37^{\circ} \mathrm{C}$. Jurkat cells were labeled with Cell Tracker Orange (Life Technologies Corporation). Next, the Jurkat T cells (ATCC) were incubated with $8 \mu \mathrm{g} / \mathrm{mL}$ camptothecin for 3 hours at $37^{\circ} \mathrm{C}, 5 \% \mathrm{CO}_{2}$ in serum-free media. At the end of co-incubation (1 hour) with apoptotic Jurkat cells, alveolar macrophages were collected 
in flow cytometry tubes and fixed in 1\% paraformaldehyde. Engulfment efficiency was measured by flow cytometry. The results were expressed as an efferocytosis index, defined as the percentage of alveolar macrophages that engulfed apoptotic cells relative to the total number of alveolar macrophages.

Apoptosis-Annexin V-FITC staining flow cytometry. Apoptosis-sensitive staining was performed as previously described $(4,51)$. Briefly, cells were labeled with Annexin V (BioLegend) and incubated for 20 minutes at RT. After washing with Annexin V binding buffer, cells were incubated for 10 minutes with LIVE/DEAD stain (Invitrogen, Thermo Fisher Scientific). Cells were washed and blocked with Fc block (CD16/32). The cells were then stained with the following fluorochrome-conjugated mouse antibodies: Ly6C-FITC (1:200, 128005, BioLegend and BD Biosciences), Gr-1-PE (1:200, 108408, BioLegend and BD Biosciences), CD11c-APCCy7 (1:200, 117324, BioLegend and BD Biosciences), F4/80-AF488 (1:200, 123120, BioLegend and BD Biosciences), and CD11b-PE-Cy7 (1:200, 101216, BioLegend and BD Biosciences). Data were analyzed using FlowJo software (Tree Star, Inc.).

Intracellular TLR 3 and dsRNA staining flow cytometry. For the detection of intracellular staining for TLR3 and dsRNA, BAL cells were collected following pneumonia. The cells were fixed, permeabilized, and subjected to intracellular staining (4). The following primary antibodies were used: J2 (1:200, 10010200, English \& Scientific Consulting), CD11c-FITC (1:200, 117306, BioLegend), and F4/80-APCCy7 (1:200, BioLegend). For TLR3 staining cells were incubated overnight at $4^{\circ} \mathrm{C}$ with polyclonal TLR3 antibody (1:1000, PA5-29619, eBioscience) and incubated with goat anti-rabbit Alexa Fluor 594 (1:1000, A-11037, BioLegend). Data were analyzed using FlowJo software.

TaqMan quantitative PCR. Total RNA was prepared from whole-lung lysates and reverse-transcribed into cDNA using M-MLV reverse transcriptase (Life Technologies Corporation). The cDNA was then amplified by real-time quantitative TaqMan PCR using an ABI Prism 7700 (Applied Biosystems) sequence detection system. GAPDH was analyzed as an internal control. TaqMan gene expression reagents or SYBR Green Master PCR mix (Applied Biosystems) were used to detect the genes responsible for inflammation. Data were expressed as the fold change in transcript expression. The fold difference in mRNA expression between treatment groups was determined by software developed by Applied Biosystems $(4,51)$.

Co-immunoprecipitation. WT mice lungs were lysed in ice-cold lysis buffer. The total protein lysate was incubated with dsRNA antibody (1:200, 10010200, English \& Scientific Consulting) for 2 hours followed by a 2-hour incubation with 20:1 of protein A-Sepharose beads (prewashed and resuspended in PBS at a 1:1 ratio). After incubation, the beads were washed 4 times with lysis buffer, separated by SDS-PAGE, transferred to PVDF membranes (MilliporeSigma), and analyzed by immunoblotting using antibodies against dsRNA and TLR3 (1:50, PA5-29619, eBioscience) (55).

Western blot/immunoblot analysis. Mice lungs were lysed in ice-cold lysis buffer, mixed with a commercial sample buffer (Invitrogen, Thermo Fisher Scientific), and heated at $95^{\circ} \mathrm{C}$ for 5 minutes. Samples were then electrophoresed on SDS-PAGE, after which the gels were transferred to PVDF membranes. Blots were incubated overnight at $4^{\circ} \mathrm{C}$ with various primary antibodies followed by the appropriate secondary antibody (118492, Jackson ImmunoResearch Laboratories). They were then washed, and the signal was detected using a Super Signal chemiluminescent substrate (Pierce Biotechnology, Thermo Fisher Scientific) $(51,56)$.

Capillary Western immunoassay. Wes analysis was performed with a Wes system (004-600, Protein Simple) according to the manufacturer's instructions using a $12-230 \mathrm{kDa}$ separation module (SM-W004, Protein Simple) and an anti-mouse detection module (DM-002, Protein Simple) (57). The samples, blocking reagent (antibody diluent), primary antibodies (1:50, Polyclonal TLR3 antibody, PA5-29619, eBioscience), HRP-conjugated secondary antibodies (1:10, Anti-Rabbit Secondary Antibody, 042-206, Protein Simple), and chemiluminescent substrate were pipetted onto the plate of the separation module.

MPO measurements. Spleen and lung sections were collected from the following groups, including WT uninjured control, WT plus KP 24 hours, and $T L R 3^{-1-}$ plus KP at 24 hours. MPO measurements were made using ELISA.

Histology. The formalin-fixed uninjured control and lung with $K P$ along with spleens were paraffin-embedded, sectioned, and stained with H\&E. Histological analysis and scoring, such as peribronchial, parenchymal, and perivascular cell infiltration, were semiquantitatively graded in a blinded manner $(18,52)$.

$T L R 3 / d s R N A$ complex inhibitor administration (in vivo). The TLR3/dsRNA complex inhibitor, (R)-2-(3Chloro-6-fluorobenzo [b] thiophene-2-carboxamido)-3-phenylpropanoic acid (Calbiochem, MilliporeSig$\mathrm{ma})$, was dissolved in DMSO and diluted in sterile PBS. The inhibitor ( $1 \mathrm{mg} / \mathrm{mouse})$ was administered i.p. to the mice 24 hours before $K P(4)$. 
Neutrophil depletion. To deplete neutrophils (in both WT and TLR $3^{-1-}$ mice), the anti-mouse Ly6G monoclonal antibody (170 $\mu \mathrm{g} /$ mouse, BP0075-1, Bio X Cell) was injected intraperitoneally, at a dose of $170 \mu \mathrm{g}$ per mouse (in $200 \mu \mathrm{L}$ ). Two days after, the whole blood was collected using potassium salt of $10 \%$ EDTA as an anticoagulant in the proportion of $50 \mu \mathrm{l}$ for every $1.0 \mathrm{~mL}$ of blood. Once obtained, whole blood was used for the complete blood cell count. Mice were inoculated with the bacterial suspension or saline control via deep oral hypopharyngeal injection under isoflurane anesthesia, and survival data were recorded every 8 hours.

Complete blood count. The analyses were performed using an automatic blood cell analyzer (HEMAVET 950).

Macrophage adoptive transfer. An age-matched group of WT and TLR $3^{-1-}$ uninjured mice alveolar macrophages were isolated from the BAL fluid. Cell suspensions were washed, RBCs were lysed, and macrophages were isolated by single-cell suspension. Macrophages were resuspended to a final density of $1 \times 10^{5}$ cells per $50 \mu \mathrm{L}$ PBS for introduction via deep oral hypopharyngeal injection under isoflurane anesthesia. After 48 hours $500 \mathrm{CFU}$ of $K P$ per $30 \mu \mathrm{L}$ of bacterial inoculum was administered. Animals were allowed to recover spontaneously, and survival data were recorded 10 days as described previously (50).

$R N A$-sequencing analysis. C57BL/6 and $T L R 3^{-1-}$ mice were subjected to a lethal model of primary insult with $K P$ administered via pharyngeal drop. Macrophages were isolated from WT and $T L R 3^{-1-}$ mice with and without injury after 24 hours $(n=4)$. Total RNA was extracted from macrophages by using the miRNeasy Mini Kit (QIAGEN) according to the manufacturer's protocol. The RNA quality was evaluated with an Agilent BioAnalyzer. All the samples had RNA integrity numbers greater than 8-9. The RNA quality was additionally assessed by electrophoresis and bioanalyzer (Agilent Technologies) before RNA-sequencing. An input of $100 \mathrm{ng}$ of total RNA was used to construct cDNA libraries (TruSeq Stranded mRNA Sample Prep Kit, Illumina) following the manufacturer's instructions. Differences between 2 groups were analyzed using Advaita. Data were deposited into the National Center for Biotechnology Information's Gene Expression Omnibus database (GSE140693).

Statistics. Data are expressed as the mean \pm SEM in all figures. Statistical significance of data between 2 groups was analyzed using 2-tailed, unpaired $t$ test with Welch's correction, and data from more than 2 groups were analyzed using 1-way ANOVA with Tukey's multiple-comparisons test (Graph Pad Prism 8.00) (4). Survival curves (Kaplan-Meier plots) were compared using log-rank tests (25). Fluorescence recovery analysis was done using ImageJ (NIH) (58). Additional methods specifically used to evaluate correlations between IHC and pneumonitis scoring for the postmortem human samples are discussed in the appropriate sections (59). Significance was set at ${ }^{*} P<0.05$; ${ }^{* *} P<0.01$; and ${ }^{* * *} P<0.001$.

Study approval. All procedures performed were approved by the Institutional Animal Care and Use Committee at the University of Michigan and complied with the state, federal, and NIH regulations. Human lung contusion specimens were obtained from the All India Institute of Medical Sciences (India), and access to these tissue specimens was approved by the local research ethics committee.

\section{Author contributions}

MVS and KR conceived and designed the research. MVS, SL, VAD, BZ, SB, SS, JM, GK, BT, MK, and AS performed research. MVS, DMA, AA, BBM, and KR performed analysis and interpretation. MVS, BBM, and $\mathrm{KR}$ drafted the manuscript for important intellectual content.

\section{Acknowledgments}

We thank Valencia Albert and Garima Agarwal, Department of Laboratory Medicine, All India Institute of Medical Sciences, New Delhi, for the preparation of human samples for TLR3 staining. We want to thank Jayakrishna Nandakumar (University of Michigan) for his expert advice and encouragement through this work and Nemanja Bratic for helping review the paper. This work was supported by NIH Grant GM111305-04 to KR.

Address correspondence to: Krishnan Raghavendran, 1500 E. Medical Center Drive, 1B407D, UH, University of Michigan, Ann Arbor, Michigan 48109, USA. Phone: 734.647.9693; Email: kraghave@umich.edu. 
1. Kochanek KD, Murphy SL, Xu J, Arias E. Mortality in the United States, 2013. NCHS Data Brief. 2014;(178):1-8.

2. Iregui M, Ward S, Sherman G, Fraser VJ, Kollef MH. Clinical importance of delays in the initiation of appropriate antibiotic treatment for ventilator-associated pneumonia. Chest. 2002;122(1):262-268.

3. Seligman R, Papassotiriou J, Morgenthaler NG, Meisner M, Teixeira PJ. Copeptin, a novel prognostic biomarker in ventilator-associated pneumonia. Crit Care. 2008;12(1):R11.

4. Suresh MV, et al. Double-Stranded RNA interacts with toll-like receptor 3 in driving the acute inflammatory response following lung contusion. Crit Care Med. 2016;44(11):e1054-e1066.

5. Dolgachev VA, Yu B, Reinke JM, Raghavendran K, Hemmila MR. Host susceptibility to gram-negative pneumonia after lung contusion. J Trauma Acute Care Surg. 2012;72(3):614-622.

6. Broug-Holub E, et al. Alveolar macrophages are required for protective pulmonary defenses in murine Klebsiella pneumonia: elimination of alveolar macrophages increases neutrophil recruitment but decreases bacterial clearance and survival. Infect Immun. 1997;65(4):1139-1146.

7. Bauer TT, Ferrer R, Angrill J, Schultze-Werninghaus G, Torres A. Ventilator-associated pneumonia: incidence, risk factors, and microbiology. Semin Respir Infect. 2000;15(4):272-279.

8. Tsan MF, Gao B. Endogenous ligands of Toll-like receptors. J Leukoc Biol. 2004;76(3):514-519.

9. Trinchieri G, Sher A. Cooperation of Toll-like receptor signals in innate immune defence. Nat Rev Immunol. 2007;7(3):179-190.

10. Ritter M, Mennerich D, Weith A, Seither P. Characterization of Toll-like receptors in primary lung epithelial cells: strong impact of the TLR3 ligand poly(I:C) on the regulation of Toll-like receptors, adaptor proteins and inflammatory response. J Inflamm (Lond). 2005;2:16.

11. Gowen BB, et al. TLR3 is essential for the induction of protective immunity against Punta Toro Virus infection by the double-stranded RNA (dsRNA), poly(I:C12U), but not poly(I:C): differential recognition of synthetic dsRNA molecules. J Immunol. 2007;178(8):5200-5208.

12. Cavassani KA, et al. TLR3 is an endogenous sensor of tissue necrosis during acute inflammatory events. J Exp Med. 2008;205(11):2609-2621.

13. Kumar H, Kawai T, Akira S. Toll-like receptors and innate immunity. Biochem Biophys Res Commun. 2009;388(4):621-625.

14. Yamamoto M, et al. Role of adaptor TRIF in the MyD88-independent toll-like receptor signaling pathway. Science. 2003;301(5633):640-643.

15. Kaiser WJ, Offermann MK. Apoptosis induced by the toll-like receptor adaptor TRIF is dependent on its receptor interacting protein homotypic interaction motif. J Immunol. 2005;174(8):4942-4952.

16. Machado-Aranda DA, Suresh MV, Yu B, Raghavendran K. Electroporation-mediated in vivo gene delivery of the Na+/K+-ATPase pump reduced lung injury in a mouse model of lung contusion. J Trauma Acute Care Surg. 2012;72(1):32-39.

17. Jiang W, Sun R, Wei H, Tian Z. Toll-like receptor 3 ligand attenuates LPS-induced liver injury by down-regulation of toll-like receptor 4 expression on macrophages. Proc Natl Acad Sci USA. 2005;102(47):17077-17082.

18. Suresh MV, et al. Pulmonary administration of a water-soluble curcumin complex reduces severity of acute lung injury. Am $J$ Respir Cell Mol Biol. 2012;47(3):280-287.

19. Sylvester I, Rankin JA, Yoshimura T, Tanaka S, Leonard EJ. Secretion of neutrophil attractant/activation protein by lipopolysaccharide-stimulated lung macrophages determined by both enzyme-linked immunosorbent assay and $\mathrm{N}$-terminal sequence analysis. Am Rev Respir Dis. 1990;141(3):683-688.

20. Alvarez D, Merino S, Tomás JM, Benedí VJ, Albertí S. Capsular polysaccharide is a major complement resistance factor in lipopolysaccharide O side chain-deficient Klebsiella pneumoniae clinical isolates. Infect Immun. 2000;68(2):953-955.

21. Regueiro V, Campos MA, Pons J, Albertí S, Bengoechea JA. The uptake of a Klebsiella pneumoniae capsule polysaccharide mutant triggers an inflammatory response by human airway epithelial cells. Microbiology (Reading, Engl). 2006;152(Pt 2):555-566.

22. Kojima Y, Weissman IL, Leeper NJ. The role of efferocytosis in atherosclerosis. Circulation. 2017;135(5):476-489.

23. Parks BW, et al. CD36, but not G2A, modulates efferocytosis, inflammation, and fibrosis following bleomycin-induced lung injury. J Lipid Res. 2013;54(4):1114-1123.

24. Sherman MA, et al. Molecular characterization of hypoxic alveolar epithelial cells after lung contusion indicates an important role for HIF-1 $\alpha$. Ann Surg. 2018;267(2):382-391.

25. Dolgachev V, et al. Electroporation-mediated delivery of FER gene enhances innate immune response and improves survival in a murine model of pneumonia. Gene Ther. 2018;25(5):359-375.

26. Berger M, et al. Neutrophils express distinct RNA receptors in a non-canonical way. J Biol Chem. 2012;287(23):19409-19417.

27. Hayashi F, Means TK, Luster AD. Toll-like receptors stimulate human neutrophil function. Blood. 2003;102(7):2660-2669.

28. Sotolongo J, Ruiz J, Fukata M. The role of innate immunity in the host defense against intestinal bacterial pathogens. Curr Infect Dis Rep. 2012;14(1):15-23.

29. Karaolis DK, et al. Cyclic di-GMP stimulates protective innate immunity in bacterial pneumonia. Infect Immun. 2007;75(10):4942-4950

30. Batra S, Cai S, Balamayooran G, Jeyaseelan S. Intrapulmonary administration of leukotriene B(4) augments neutrophil accumulation and responses in the lung to Klebsiella infection in CXCL1 knockout mice. J Immunol. 2012;188(7):3458-3468.

31. Khoubnasabjafari M, Ansarin K, Jouyban A. Reliability of malondialdehyde as a biomarker of oxidative stress in psychological disorders. Bioimpacts. 2015;5(3):123-127.

32. Reber LL, et al. Neutrophil myeloperoxidase diminishes the toxic effects and mortality induced by lipopolysaccharide. $J$ Exp Med. 2017;214(5):1249-1258.

33. DePalma RG, Burris DG, Champion HR, Hodgson MJ. Blast injuries. N Engl J Med. 2005;352(13):1335-1342.

34. Miller PR, Croce MA, Kilgo PD, Scott J, Fabian TC. Acute respiratory distress syndrome in blunt trauma: identification of independent risk factors. Am Surg. 2002;68(10):845-850.

35. Miller PR, et al. ARDS after pulmonary contusion: accurate measurement of contusion volume identifies high-risk patients J Trauma. 2001;51(2):223-228.

36. Pan ZK, Fisher C, Li JD, Jiang Y, Huang S, Chen LY. Bacterial LPS up-regulated TLR3 expression is critical for antiviral response in human monocytes: evidence for negative regulation by CYLD. Int Immunol. 2011;23(6):357-364. 
37. Hoth JJ, et al. Toll-like receptor 2 participates in the response to lung injury in a murine model of pulmonary contusion. Shock. 2007;28(4):447-452.

38. Fisher BD, Britman NA, Null RH. Staphylococcal toxic shock syndrome following herniorrhaphy in a male. $J M e d$ Soc $N J$. 1982;79(12):925-926.

39. Muzio M, et al. Differential expression and regulation of toll-like receptors (TLR) in human leukocytes: selective expression of TLR3 in dendritic cells. J Immunol. 2000;164(11):5998-6004.

40. Sabroe I, et al. Selective roles for Toll-like receptor (TLR)2 and TLR4 in the regulation of neutrophil activation and life span. J Immunol. 2003;170(10):5268-5275.

41. Chun CD, Liles WC, Frevert CW, Glenny RW, Altemeier WA. Mechanical ventilation modulates Toll-like receptor-3-induced lung inflammation via a MyD88-dependent, TLR4-independent pathway: a controlled animal study. BMC Pulm Med. $2010 ; 10: 57$.

42. O'Neill LA, Bryant CE, Doyle SL. Therapeutic targeting of Toll-like receptors for infectious and inflammatory diseases and cancer. Pharmacol Rev. 2009;61(2):177-197.

43. Dockrell DH, et al. Alveolar macrophage apoptosis contributes to pneumococcal clearance in a resolving model of pulmonary infection. J Immunol. 2003;171(10):5380-5388.

44. Highlander SK, Fedorova ND, Dusek DM, Panciera R, Alvarez LE, Rinehart C. Inactivation of Pasteurella (Mannheimia) haemolytica leukotoxin causes partial attenuation of virulence in a calf challenge model. Infect Immun. 2000;68(7):3916-3922.

45. Serezani CH, Aronoff DM, Jancar S, Mancuso P, Peters-Golden M. Leukotrienes enhance the bactericidal activity of alveolar macrophages against Klebsiella pneumoniae through the activation of NADPH oxidase. Blood. 2005;106(3):1067-1075.

46. Domingo-Gonzalez R, Moore BB. Defective pulmonary innate immune responses post-stem cell transplantation; review and results from one model system. Front Immunol. 2013;4:126.

47. Baral P, Batra S, Zemans RL, Downey GP, Jeyaseelan S. Divergent functions of Toll-like receptors during bacterial lung infections. Am J Respir Crit Care Med. 2014;190(7):722-732.

48. Suresh MV, et al. The protective role of MnTBAP in oxidant-mediated injury and inflammation in a rat model of lung contusion. Surgery. 2013;154(5):980-990.

49. Obi AT, et al. Gram-Negative pneumonia alters large-vein cell-adhesion molecule profile and potentiates experimental stasis venous thrombosis. J Vasc Res. 2016;53(3-4):186-195.

50. Cai S, Batra S, Del Piero F, Jeyaseelan S. NLRP12 modulates host defense through IL-17A-CXCL1 axis. Mucosal Immunol. 2016;9(2):503-514.

51. Suresh MV, et al. Activation of hypoxia-inducible factor- $1 \alpha$ in type 2 alveolar epithelial cell is a major driver of acute inflammation following lung contusion. Crit Care Med. 2014;42(10):e642-e653.

52. Suresh MV, et al. Role of macrophage chemoattractant protein-1 in acute inflammation after lung contusion. Am J Respir Cell Mol Biol. 2012;46(6):797-806.

53. Domingo-Gonzalez R, Katz S, Serezani CH, Moore TA, Levine AM, Moore BB. Prostaglandin E2-induced changes in alveolar macrophage scavenger receptor profiles differentially alter phagocytosis of Pseudomonas aeruginosa and Staphylococcus aureus post-bone marrow transplant. J Immunol. 2013;190(11):5809-5817.

54. Serban KA, et al. Alpha-1 antitrypsin supplementation improves alveolar macrophages efferocytosis and phagocytosis following cigarette smoke exposure. PLoS ONE. 2017;12(4):e0176073.

55. Aschkenasy G, Bromberg Z, Raj N, Deutschman CS, Weiss YG. Enhanced Hsp70 expression protects against acute lung injury by modulating apoptotic pathways. PLoS ONE. 2011;6(11):e26956.

56. Ghimire L, Paudel S, Jin L, Baral P, Cai S, Jeyaseelan S. NLRP6 negatively regulates pulmonary host defense in Gram-positive bacterial infection through modulating neutrophil recruitment and function. PLoS Pathog. 2018;14(9):e1007308.

57. Beekman C, Janson AA, Baghat A, van Deutekom JC, Datson NA. Use of capillary Western immunoassay (Wes) for quantification of dystrophin levels in skeletal muscle of healthy controls and individuals with Becker and Duchenne muscular dystrophy. PLoS ONE. 2018;13(4):e0195850

58. Zheng CY, Petralia RS, Wang YX, Kachar B. Fluorescence recovery after photobleaching (FRAP) of fluorescence tagged proteins in dendritic spines of cultured hippocampal neurons. J Vis Exp. 2011;(50):2568.

59. Vasakova M, Morell F, Walsh S, Leslie K, Raghu G. Hypersensitivity pneumonitis: perspectives in diagnosis and management. Am J Respir Crit Care Med. 2017;196(6):680-689. 